\title{
Generation of class fields by the modular function $j_{1,12}$
}

\author{
by \\ Kuk Jin Hong and Ja Kyung Koo (Taejon) \\ Dedicated to Professor Takashi Ono \\ on the occasion of his 70th birthday
}

1. Introduction. Let $\mathfrak{H}$ be the complex upper half plane and let $\Gamma$ be a congruence subgroup of $\mathrm{SL}_{2}(\mathbb{Z})$. Since the group $\Gamma$ acts on $\mathfrak{H}$ by linear fractional transformations, we get the modular curve $X(\Gamma)=\Gamma \backslash \mathfrak{H}^{*}$, as the projective closure of smooth affine curve $\Gamma \backslash \mathfrak{H}$, with genus $g_{\Gamma}$. Since $g_{1, N}=0$ only for the eleven cases $1 \leq N \leq 10$ and $N=12([12])$ when $\Gamma=\Gamma_{1}(N)$ $\left(=\left\{\gamma \in \mathrm{SL}_{2}(\mathbb{Z}) \mid \gamma \equiv\left(\begin{array}{ll}1 & * \\ 0 & 1\end{array}\right)(\bmod N)\right\}\right)$, the function field $K\left(X_{1}(12)\right)$ over the curve $X_{1}(12)=\Gamma_{1}(12) \backslash \mathfrak{H}^{*}$ is a rational function field $\mathbb{C}\left(j_{1,12}\right)$ where $j_{1,12}(z):=\theta_{3}(2 z) / \theta_{3}(6 z)$ for $z \in \mathfrak{H}$ and $\theta_{3}$ is the classical Jacobi theta series.

In this article we will construct in Section 3 some sort of class fields by means of Shimura's ideas for the congruence subgroups $\Gamma(N), \Gamma_{0}(N)$ and $\Gamma_{1}(N)$. In Section 4 we will generate the ray class field $K_{(12)}$ with conductor 12 of imaginary quadratic fields $K$ by applying standard results of complex multiplication to the modular function $j_{1,12}(z)$. In Section 5 by using Chen-Yui's result [1], we shall investigate when the subfield of $K_{(12)}$ generated by $j_{1,12}(\alpha)$ is equal to a ray class field $K_{\mathfrak{f}}$ for a conductor $\mathfrak{f}$ dividing 12 where $\alpha$ is the quotient of a basis of an $\mathcal{O}_{K}$-ideal (Theorems 20, 21 and 23). Lastly, in Section 6 we will explore an explicit formula for the conjugates of the Hauptmodul $N\left(j_{1,12}(\alpha)\right)$ permitting the numerical computation of its minimal polynomial. We thank the referee for his valuable comments which enabled us to improve Sections 5 and 6 .

Throughout the article we adopt the following notations:

- $\Gamma(N)=\left\{\gamma \in \mathrm{SL}_{2}(\mathbb{Z}) \mid \gamma \equiv I(\bmod N)\right\}$,

- $\Gamma_{0}(N)=\left\{\left(\begin{array}{ll}a & b \\ c & d\end{array}\right) \in \Gamma(1) \mid c \equiv 0(\bmod N)\right\}$

- $\Gamma^{1}(N)=\left\{\left(\begin{array}{ll}a & b \\ c & d\end{array}\right) \in \Gamma(1) \mid a \equiv d \equiv 1, b \equiv 0(\bmod N)\right\}$,

2000 Mathematics Subject Classification: 11F11, 11R04, 11R37, $14 \mathrm{H} 55$.

This article was supported by KOSEF 98-0701-01-01-3. 
- $\Gamma_{0}(N, M)=\left\{\left(\begin{array}{ll}a & b \\ c & d\end{array}\right) \in \Gamma(1) \mid b \equiv 0(\bmod M), c \equiv 0(\bmod N)\right\}$,

- $M_{k / 2}\left(\widetilde{\Gamma}_{0}(N)\right)$, the space of modular forms of half integral weight for the group $\Gamma_{0}(N)$,

- $M_{k / 2}\left(\widetilde{\Gamma}_{0}(N), \chi\right)=\left\{f \in M_{k / 2}\left(\widetilde{\Gamma}_{0}(N)\right) \mid f(\gamma z)=\chi(d) j(\gamma, z)^{k} f(z)\right.$ for all $\left.\gamma=\left(\begin{array}{ll}* & * \\ c & d\end{array}\right) \in \Gamma_{0}(N)\right\}$ where $\chi$ is a Dirichlet character modulo $N$ and $j(\gamma, z)=(c / d) \varepsilon_{d}^{-1} \sqrt{c z+d}$ with $\varepsilon_{d}=1$ if $d \equiv 1(\bmod 4)$ and $=i$ otherwise,

- $\mathbb{Z}_{p}$, the ring of $p$-adic integers,

- $\mathbb{Q}_{p}$, the field of $p$-adic numbers,

- $q_{h}=e^{2 \pi i z / h}, z \in \mathfrak{H}$.

2. Hauptmodul of $K\left(X_{1}(12)\right)$ as a quotient of Jacobi theta series. For $\mu, \nu \in \mathbb{R}$ and $z \in \mathfrak{H}$, put

$$
\Theta_{\mu, \nu}(z):=\sum_{n \in \mathbb{Z}} \exp \left\{\pi i\left(n+\frac{1}{2} \mu\right)^{2} z+\pi i n \nu\right\} .
$$

This series converges uniformly for $\operatorname{Im}(z) \geq \eta>0$, and hence defines a holomorphic function on $\mathfrak{H}$. Then the Jacobi theta series $\theta_{2}, \theta_{3}$ and $\theta_{4}$ are defined by

$$
\begin{aligned}
& \theta_{2}(z):=\Theta_{1,0}(z)=\sum_{n \in \mathbb{Z}} q_{2}^{(n+1 / 2)^{2}}, \\
& \theta_{3}(z):=\Theta_{0,0}(z)=\sum_{n \in \mathbb{Z}} q_{2}^{n^{2}}, \\
& \theta_{4}(z):=\Theta_{0,1}(z)=\sum_{n \in \mathbb{Z}}(-1)^{n} q_{2}^{n^{2}} .
\end{aligned}
$$

And we have the following transformation formulas ([17], pp. 218-219):

$$
\begin{array}{ll}
\theta_{2}(z+1)=e^{\pi i / 4} \theta_{2}(z), & \theta_{2}(-1 / z)=(-i z)^{1 / 2} \theta_{4}(z), \\
\theta_{3}(z+1)=\theta_{4}(z), & \theta_{3}(-1 / z)=(-i z)^{1 / 2} \theta_{3}(z), \\
\theta_{4}(z+1)=\theta_{3}(z), & \theta_{4}(-1 / z)=(-i z)^{1 / 2} \theta_{2}(z) .
\end{array}
$$

Furthermore, we have the following theorem at hand. For the definition of modular forms of half integer weight, we refer to [20] or [14].

TheOREM 1. (1) $\theta_{3}(2 z) \in M_{1 / 2}\left(\widetilde{\Gamma}_{0}(4)\right)$ and $\theta_{3}(6 z) \in M_{1 / 2}\left(\widetilde{\Gamma}_{0}(12), \chi_{3}\right)$.

(2) $K\left(X_{1}(12)\right)=\mathbb{C}\left(j_{1,12}\right)$ and $j_{1,12}$ takes the following value at each cusp: $j_{1,12}(\infty)=1, j_{1,12}(0)=\sqrt{3}, j_{1,12}(1 / 2)=0$ (a simple zero), $j_{1,12}(1 / 3)$ $=i, j_{1,12}(1 / 4)=\sqrt{3} i, j_{1,12}(1 / 5)=-\sqrt{3}, j_{1,12}(1 / 6)=\infty$ (a simple pole $)$, $j_{1,12}(1 / 8)=-\sqrt{3} i, j_{1,12}(1 / 9)=-i, j_{1,12}(5 / 12)=-1$.

Proof. [11], Theorem 4. 
3. Generation I. Let $\Gamma$ be a Fuchsian group of the first kind. Then $\Gamma \backslash \mathfrak{H}^{*}(=X(\Gamma))$ is a compact Riemann surface. Hence, there exists a projective nonsingular algebraic curve $V_{\Gamma}$, defined over $\mathbb{C}$, biregularly isomorphic to $\Gamma \backslash \mathfrak{H}^{*}$. We specify a $\Gamma$-invariant holomorphic map $\varphi_{\Gamma}$ of $\mathfrak{H}^{*}$ to $V_{\Gamma}$ which gives a biregular isomorphism of $\Gamma \backslash \mathfrak{H}^{*}$ to $V_{\Gamma}$. In that situation, we call $\left(V_{\Gamma}, \varphi_{\Gamma}\right)$ a model of $\Gamma \backslash \mathfrak{H}^{*}$. Through this article we always assume that the genus of $\Gamma \backslash \mathfrak{H}^{*}$ is zero. Then its function field $K(X(\Gamma))$ is equal to $\mathbb{C}\left(J^{\prime}\right)$ for some $J^{\prime} \in K(X(\Gamma))$.

Lemma 2. $\left(\mathbb{P}^{1}(\mathbb{C}), J^{\prime}\right)$ is a model of $\Gamma \backslash \mathfrak{H}^{*}$.

Proof. [6], Lemma 14.

Let $G_{\mathbb{A}}$ be the adelization of an algebraic group $G=\mathrm{GL}_{2}$ defined over Q. Put

$$
\begin{aligned}
G_{p} & =\mathrm{GL}_{2}\left(\mathbb{Q}_{p}\right) \quad(p \text { a rational prime }) \\
G_{\infty} & =\mathrm{GL}_{2}(\mathbb{R}), \\
G_{\infty+} & =\left\{x \in G_{\infty} \mid \operatorname{det}(x)>0\right\} \\
G_{\mathbb{Q}+} & =\left\{x \in \mathrm{GL}_{2}(\mathbb{Q}) \mid \operatorname{det}(x)>0\right\} .
\end{aligned}
$$

We define the topology of $G_{\mathbb{A}}$ by taking $U=\prod_{p} \mathrm{GL}_{2}\left(\mathbb{Z}_{p}\right) \times G_{\infty+}$ to be an open subgroup of $G_{\mathbb{A}}$. Let $K$ be an imaginary quadratic field and $\xi$ be an embedding of $K$ into $M_{2}(\mathbb{Q})$. We call $\xi$ normalized if it is defined by

$$
a\left(\begin{array}{c}
z \\
1
\end{array}\right)=\xi(a)\left(\begin{array}{l}
z \\
1
\end{array}\right) \quad \text { for } a \in K
$$

where $z$ is the fixed point of $\xi\left(K^{\times}\right)\left(\subset G_{\mathbb{Q}+}\right)$ in $\mathfrak{H}$. Observe that the embedding $\xi$ defines a continuous homomorphism of $K_{\mathbb{A}}^{\times}$into $G_{\mathbb{A}+}$, which we denote again by $\xi$. Here $G_{\mathbb{A}+}$ is the group $G_{0} G_{\infty+}$ with $G_{0}$ the nonarchimedean part of $G_{\mathbb{A}}$ and $K_{\mathbb{A}}^{\times}$is the idele group of $K$.

Let $\mathcal{Z}$ be the set of open subgroups $S$ of $G_{\mathbb{A}+}$ containing $\mathbb{Q}^{\times} G_{\infty+}$ such that $S / \mathbb{Q}^{\times} G_{\infty+}$ is compact. For $S \in \mathcal{Z}$, we see that $\operatorname{det}(S)$ is open in $\mathbb{Q}_{\mathbb{A}}^{\times}$. Therefore the subgroup $\mathbb{Q}^{\times} \cdot \operatorname{det}(S)$ of $\mathbb{Q}_{\mathbb{A}}^{\times}$corresponds to a finite abelian extension of $\mathbb{Q}$, which we write $k_{S}$. Put $\Gamma_{S}=S \cap G_{\mathbb{Q}+}$ for $S \in \mathcal{Z}$. As is well known ([19], Proposition 6.27), $\Gamma_{S} / \mathbb{Q}^{\times}$is a Fuchsian group of the first kind commensurable with $\Gamma(1) /\{ \pm 1\}$.

Proposition 3. Let $\Gamma^{\prime}$ be a discrete subgroup of $G_{\infty+} / \mathbb{R}^{\times}$commensurable with $\mathbb{Q}^{\times} \Gamma(1) / \mathbb{Q}^{\times}$, and containing $\Gamma(N)$ for some $N$. Then $\Gamma^{\prime}=$ $\Gamma_{S} / \mathbb{Q}^{\times}$for some $S \in \mathcal{Z}$.

Pr o of. [19], Proposition 6.30.

In accordance with Proposition 3, we are able to find open compact subgroups $S$ corresponding to $\Gamma_{0}(N), \Gamma_{0}(N, M), \Gamma_{1}(N)$ and $\Gamma^{1}(N)$. Fix 
positive integers $N$ and $M$, and consider the following:

$$
\begin{aligned}
U_{(p)} & =\left\{\left(\begin{array}{ll}
a & b \\
c & d
\end{array}\right) \in \mathrm{GL}_{2}\left(\mathbb{Z}_{p}\right) \mid\left(\begin{array}{ll}
a & b \\
c & d
\end{array}\right) \equiv\left(\begin{array}{ll}
1 & 0 \\
0 & 1
\end{array}\right)\left(\bmod N \mathbb{Z}_{p}\right)\right\}, \\
U_{0,(p)} & =\left\{\left(\begin{array}{ll}
a & b \\
c & d
\end{array}\right) \in \mathrm{GL}_{2}\left(\mathbb{Z}_{p}\right) \mid c \equiv 0\left(\bmod N \mathbb{Z}_{p}\right)\right\}, \\
U_{0,(p)}^{0} & =\left\{\left(\begin{array}{ll}
a & b \\
c & d
\end{array}\right) \in \mathrm{GL}_{2}\left(\mathbb{Z}_{p}\right) \mid b \equiv 0\left(\bmod M \mathbb{Z}_{p}\right), c \equiv 0\left(\bmod N \mathbb{Z}_{p}\right)\right\}, \\
U_{1,(p)} & =\left\{\left(\begin{array}{ll}
a & b \\
c & d
\end{array}\right) \in \mathrm{GL}_{2}\left(\mathbb{Z}_{p}\right) \mid a \equiv d \equiv 1, c \equiv 0\left(\bmod N \mathbb{Z}_{p}\right)\right\}, \\
U_{(p)}^{1} & =\left\{\left(\begin{array}{ll}
a & b \\
c & d
\end{array}\right) \in \mathrm{GL}_{2}\left(\mathbb{Z}_{p}\right) \mid a \equiv d \equiv 1, b \equiv 0\left(\bmod N \mathbb{Z}_{p}\right)\right\}, \\
U_{N} & =\left\{x=\left(x_{p}\right) \in U \mid x_{p} \in U_{(p)} \text { for all finite } p\right\}, \\
U_{0} & =\left\{x=\left(x_{p}\right) \in U \mid x_{p} \in U_{0,(p)} \text { for all finite } p\right\}, \\
U_{0}^{0} & =\left\{x=\left(x_{p}\right) \in U \mid x_{p} \in U_{0,(p)}^{0} \text { for all finite } p\right\}, \\
U_{1} & =\left\{x=\left(x_{p}\right) \in U \mid x_{p} \in U_{1,(p)} \text { for all finite } p\right\}, \\
U^{1} & =\left\{x=\left(x_{p}\right) \in U \mid x_{p} \in U_{(p)}^{1} \text { for all finite } p\right\} .
\end{aligned}
$$

Put

$$
S=\mathbb{Q}^{\times} U_{N}, \quad S_{0}=\mathbb{Q}^{\times} U_{0}, \quad S_{0}^{0}=\mathbb{Q}^{\times} U_{0}^{0}, \quad S_{1}=\mathbb{Q}^{\times} U_{1}, \quad S^{1}=\mathbb{Q}^{\times} U^{1} .
$$

We then have the following lemmas.

LeMmA 4. (i) $S_{0}, S_{0}^{0} \in \mathcal{Z}$.

(ii) $k_{S_{0}}=k_{S_{0}^{0}}=\mathbb{Q}$.

(iii) $\Gamma_{S_{0}}=\mathbb{Q}^{\times} \Gamma_{0}(N)$ and $\Gamma_{S_{0}^{0}}=\mathbb{Q}^{\times} \Gamma_{0}(N, M)$.

Pr o of. First, we observe that $\mathbb{Q}^{\times} U_{0}$ (resp. $\mathbb{Q}^{\times} U_{0}^{0}$ ) is an open subgroup of $\mathbb{Q}^{\times} U$ since $\mathbb{Q}^{\times} U_{0}$ (resp. $\left.\mathbb{Q}^{\times} U_{0}^{0}\right)$ contains $\mathbb{Q}^{\times} U_{N}$ (resp. $\mathbb{Q}^{\times} U_{\text {l.c.m. }\{N, M\}}$ ). Hence, for (i), it is enough to show that $\mathbb{Q}^{\times} U / \mathbb{Q}^{\times} G_{\infty}+$ is compact. But, we know that $\mathbb{Q}^{\times} U / \mathbb{Q}^{\times} G_{\infty+}=\prod_{p} \mathrm{GL}_{2}\left(\mathbb{Z}_{p}\right)$ is compact because each $\mathrm{GL}_{2}\left(\mathbb{Z}_{p}\right)$ is a profinite group. For (ii), note by class field theory that $\mathbb{Q}$ corresponds to the norm group $\mathbb{Q}^{\times} \cdot \mathbb{Q}_{\mathbb{A}}^{\times \infty}$ with $\mathbb{Q}_{\mathbb{A}}^{\times \infty}=\mathbb{R}^{\times} \times \prod_{p} \mathbb{Z}_{p}^{\times}$.

We claim that $\operatorname{det}\left(U_{0}\right)=\operatorname{det}\left(U_{0}^{0}\right)=\mathbb{Q}_{\mathbb{A}}^{\times \infty}$. Indeed, it is obvious that $\operatorname{det}\left(U_{0}\right), \operatorname{det}\left(U_{0}^{0}\right) \subset \mathbb{Q}_{\mathbb{A}}^{\times \infty}$. Conversely, for any element $\left(\alpha_{p}\right) \in \mathbb{Q}_{\mathbb{A}}^{\times \infty}$, take $y_{p}=\left(\begin{array}{cc}1 & 0 \\ 0 & \alpha_{p}\end{array}\right)$. Then $\left(y_{p}\right) \in U_{0}, U_{0}^{0}$ and $\operatorname{det}\left(y_{p}\right)=\left(\operatorname{det} y_{p}\right)=\left(\alpha_{p}\right)$. Finally, we come up with $\Gamma_{S_{0}}=\mathbb{Q}^{\times} U_{0} \cap G_{\mathbb{Q}+}=\mathbb{Q}^{\times}\left(U_{0} \cap G_{\mathbb{Q}+}\right)=\mathbb{Q}^{\times} \Gamma_{0}(N)$ and $\Gamma_{S_{0}^{0}}=\mathbb{Q}^{\times} U_{0}^{0} \cap G_{\mathbb{Q}+}=\mathbb{Q}^{\times}\left(U_{0}^{0} \cap G_{\mathbb{Q}+}\right)=\mathbb{Q}^{\times} \Gamma_{0}(N, M)$.

Lemma 5. (i) $S_{1}, S^{1} \in \mathcal{Z}$.

(ii) $k_{S_{1}}=k_{S^{1}}=\mathbb{Q}\left(\zeta_{N}\right)$ where $\zeta_{N}=e^{2 \pi i / N}$.

(iii) $\Gamma_{S_{1}}=\mathbb{Q}^{\times} \Gamma_{1}(N)$ and $\Gamma_{S^{1}}=\mathbb{Q}^{\times} \Gamma^{1}(N)$.

Proof. (i) follows from the same method as in Lemma 4(i). Let

$$
V_{N p_{\infty}}=\left\{\alpha=\left(\alpha_{p}\right) \in \mathbb{Q}_{\mathbb{A}}^{\times} \mid \alpha \equiv 1\left(\bmod ^{*} N p_{\infty}\right), \alpha_{p} \in \mathbb{Z}_{p}^{\times} \text {for } p \nmid N\right\}
$$


where $p_{\infty}$ denotes the infinite $\mathbb{Q}$-prime. Here $\alpha \equiv 1\left(\bmod ^{*} N p_{\infty}\right)$ means that each $\alpha_{p_{i}}$ is congruent to $1\left(\bmod p_{i}^{n_{i}} \mathbb{Z}_{p_{i}}\right)$ if $N=p_{1}^{n_{1}} \ldots p_{r}^{n_{r}}$ and $\alpha_{p_{\infty}}>0$. As is well known $([15]$, p. 209$), \mathbb{Q}\left(\zeta_{N}\right)$ is the class field corresponding to $\mathbb{Q}^{\times} V_{N p_{\infty}}$.

Now as for (ii), it suffices to show that $\operatorname{det}\left(U_{1}\right)=\operatorname{det}\left(U^{1}\right)=V_{N p_{\infty}}$. For $\left(x_{p}\right) \in U_{1}, U^{1}, \operatorname{det}\left(x_{p}\right) \equiv 1\left(\bmod N \mathbb{Z}_{p}\right) \equiv 1\left(\bmod p^{n} \mathbb{Z}_{p}\right)$ when $p^{n} \| N$. Hence, $\operatorname{det}\left(U_{1}\right), \operatorname{det}\left(U^{1}\right) \subset V_{N p_{\infty}}$. Conversely, for $\left(\alpha_{p}\right) \in V_{N p_{\infty}}$, take $x_{p}=$ $\left(\begin{array}{ll}1 & 0 \\ 0 & \alpha_{p}\end{array}\right)$. Since $N \mathbb{Z}_{p}=p^{n} \mathbb{Z}_{p}$ and $\alpha_{p} \equiv 1\left(\bmod p^{n} \mathbb{Z}_{p}\right)$ for $p^{n} \| N$, it is clear that $\left(x_{p}\right) \in U_{1}, U^{1}$ and $\operatorname{det}\left(x_{p}\right)=\alpha_{p}$. Finally, we end up with $\Gamma_{S_{1}}=$ $\mathbb{Q}^{\times} U_{1} \cap G_{\mathbb{Q}+}=\mathbb{Q}^{\times}\left(U_{1} \cap G_{\mathbb{Q}+}\right)=\mathbb{Q}^{\times} \Gamma_{1}(N)$ and $\Gamma_{S^{1}}=\mathbb{Q}^{\times} U^{1} \cap G_{\mathbb{Q}+}=$ $\mathbb{Q}^{\times}\left(U^{1} \cap G_{\mathbb{Q}+}\right)=\mathbb{Q}^{\times} \Gamma^{1}(N)$.

REMARK 6. Now we consider a normalized embedding $\xi_{z}: K \rightarrow M_{2}(\mathbb{Q})$ defined by $a\left(\begin{array}{l}z \\ 1\end{array}\right)=\xi_{z}(a)\left(\begin{array}{l}z \\ 1\end{array}\right)$ for $a \in K$ and $z \in K \cap \mathfrak{H}$. Then $z$ is the fixed point of $\xi\left(K^{\times}\right)$in $\mathfrak{H}$. Let $\left(V_{T}, \varphi_{T}\right)$ be a model of $\Gamma_{T} \backslash \mathfrak{H}^{*}$ for $T \in$ $\left\{S_{0}, S_{0}^{0}, S_{1}, S^{1}\right\}$. Note that, for convenience, we identify $V_{T}$ and $\varphi_{T}$ with a projective nonsingular algebraic curve $V_{\Gamma_{T}}$ and a $\Gamma_{T}$-invariant holomorphic map $\varphi_{\Gamma_{T}}$, respectively.

We see by [4] that $\varphi_{S_{0}}$ can be chosen as the product of Dedekind eta functions and $V_{S_{0}}=\mathbb{P}^{1}(\mathbb{C})$. It then follows from [19], Proposition 6.31, that $\varphi_{S_{0}}(z)$ belongs to $\mathbb{P}^{1}\left(K^{\mathrm{ab}}\right)$ for the curves $X_{0}(N)=\Gamma_{0}(N) \backslash \mathfrak{H}^{*}$ where $K^{\mathrm{ab}}$ is the maximal abelian extension of $K$. Furthermore, it is true that the Dedekind eta function $\eta(z)$ has no zeros in $\mathfrak{H}$. Hence we conclude that $\varphi_{S_{0}}(z)$ in fact belongs to $K^{\text {ab }}$ for $z \in K \cap \mathfrak{H}$. On the other hand, since $\left(\begin{array}{cc}M & 0 \\ 0 & 1\end{array}\right)^{-1} \Gamma_{0}(N, M)\left(\begin{array}{cc}M & 0 \\ 0 & 1\end{array}\right)=\Gamma_{0}(N M)$, two modular curves $X_{0}(N, M)=$ $\Gamma_{0}(N, M) \backslash \mathfrak{H}^{*}$ and $X_{0}(N M)=\Gamma_{0}(N M) \backslash \mathfrak{H}^{*}$ are isomorphic and hence the genera of $X_{0}(N, M)$ are completely determined by those of $X_{0}(N M)$, and vice versa.

We recall from [19], Section 6.7, the following general situation.

Let $\Gamma^{\prime}$ be another Fuchsian group of the first kind, $\mathfrak{H}^{* \prime}$ the union of $\mathfrak{H}$ and the cusps of $\Gamma^{\prime}$, and $\left(V_{\Gamma^{\prime}}, \varphi_{\Gamma^{\prime}}\right)$ a model of $\Gamma^{\prime} \backslash \mathfrak{H}^{* \prime}$. Suppose that $\alpha \Gamma \alpha^{-1} \subset \Gamma^{\prime}$ with an element $\alpha$ in $G_{\infty+}$. Then we can define a rational map $T$ of $V_{\Gamma}$ to $V_{\Gamma^{\prime}}$ by $T\left(\varphi_{\Gamma}(z)\right)=\varphi_{\Gamma^{\prime}}(\alpha(z))$, that is, by the following commutative diagram:

$$
\begin{array}{rlll}
\mathfrak{H}^{*} & \stackrel{\alpha}{\rightarrow} & \mathfrak{H}^{* \prime} \\
\varphi_{\Gamma} \downarrow & & \downarrow \varphi_{\Gamma^{\prime}} \\
V_{\Gamma} & \stackrel{T}{\rightarrow} & V_{\Gamma^{\prime}}
\end{array}
$$

This includes, as special cases, the following two types of maps:

CAse (a): $\alpha=1$, hence $\Gamma \subset \Gamma^{\prime}$. Then $T$ is the usual projection map. CASE (b): $\alpha \Gamma \alpha^{-1}=\Gamma^{\prime}$. Then $T$ is a biregular isomorphism of $V_{\Gamma}$ to $V_{\Gamma^{\prime}}$. 
We shall apply our situation to Case (b). Take $\Gamma=\Gamma_{0}(N, M), \Gamma^{\prime}=$ $\Gamma_{0}(N M)$ and $\alpha=\left(\begin{array}{cc}M & 0 \\ 0 & 1\end{array}\right)^{-1}$. Then we have $T\left(\varphi_{\Gamma_{0}(N, M)}(z)\right)=\varphi_{\Gamma_{0}(N M)}(\alpha(z))$, which means that $\left(\mathbb{P}^{1}(\mathbb{C}), \varphi_{\Gamma_{0}(N M)}(z / M)\right)$ is a model of $\Gamma_{0}(N, M) \backslash \mathfrak{H}^{*}$. In particular, since the genera of $\Gamma_{0}(N M) \backslash \mathfrak{H}^{*}$ and $\Gamma_{0}(N, M) \backslash \mathfrak{H}^{*}$ are all zeros, we can take $\varphi_{\Gamma_{0}(N M)}(z)$ and $\varphi_{\Gamma_{0}(N M)}(z / M)$ as Hauptmoduln. Therefore we can construct the following class fields by making use of the Hauptmoduln of genus zero curves $X_{0}(N)$. We refer to the Appendix for those Hauptmoduln.

TheOREM 7. Let $K$ be an imaginary quadratic field and let $\xi_{z}$ be the normalized embedding for fixed $z \in K \cap \mathfrak{H}$. Then $\varphi_{S_{0}}(z)$ belongs to the maximal abelian extension $K^{\mathrm{ab}}$ of $K$ and $K\left(\varphi_{S_{0}}(z)\right)$ is the class field of $K$ corresponding to the subgroup $K^{\times} \cdot \xi_{z}^{-1}\left(S_{0}\right)$ of $K_{\mathbb{A}}^{\times}$.

Proof. In the case of $S_{0}$, we have $k_{S_{0}}=\mathbb{Q}$ and $\Gamma_{S_{0}}=\mathbb{Q}^{\times} \Gamma_{0}(N)$ by Lemma 4(ii) and (iii). Since $\varphi_{S_{0}}$ gives a model of the curve $X_{0}(N)$, the assertion follows from [19], Proposition 6.33, and Remark 6.

Since $\left(\begin{array}{cc}M & 0 \\ 0 & 1\end{array}\right) \xi_{z / M}(a)\left(\begin{array}{cc}M & 0 \\ 0 & 1\end{array}\right)^{-1}=\xi_{z}(a)$ for $a \in K$,

$$
K^{\times} \cdot \xi_{z / M}^{-1}\left(\mathbb{Q}^{\times} U_{0}\right)=K^{\times} \cdot \xi_{z}^{-1}\left(\mathbb{Q}^{\times} U_{0}^{0}\right)
$$

and hence we have the following corollary for $\Gamma_{0}(N, M)$.

Corollary 8. Notations being as in Theorem $7, \varphi_{S_{0}}(z / M)$ is in the maximal abelian extension $K^{\mathrm{ab}}$ of $K$ when $g_{\Gamma_{0}(N, M)}=0$ and $K\left(\varphi_{S_{0}}(z / M)\right)$ is the class field of $K$ corresponding to the subgroup $K^{\times} \cdot \xi_{z}^{-1}\left(S_{0}^{0}\right)$ of $K_{\mathbb{A}}^{\times}$.

We refer to the Appendix for the Hauptmoduln of genus zero curves $X(N)$ (except for the case $N=5$ ) and $X_{1}(N)$. Again by [19], Proposition 6.31, each Hauptmodul listed in Table 4 belongs to $\mathbb{P}^{1}\left(K^{\mathrm{ab}}\right)$. Since the Hauptmoduln have poles only at $\infty$, we see that they in fact take values in $K^{\mathrm{ab}}$ for $z \in K \cap \mathfrak{H}$. As an analogue of Theorem 7 in the case of $\Gamma(N)$ $(N=2,3,4)$ and $\Gamma_{1}(N)(1 \leq N \leq 10$ and $N=12)$, we get the following theorem.

Theorem 9. Let $K$ be an imaginary quadratic field and let $\xi_{z}$ be the normalized embedding for $z \in K \cap \mathfrak{H}$. Then $N\left(j_{1, N}(z)\right)$ and $N\left(j_{N}(z)\right)$ belong to the maximal abelian extension $K^{\mathrm{ab}}$ of $K$ and $K\left(N\left(j_{1, N}(z)\right), \zeta_{N}\right)$ (resp. $\left.K\left(N\left(j_{N}(z)\right), \zeta_{N}\right)\right)$ is the class field of $K$ corresponding to the subgroup $K^{\times} \cdot \xi_{z}^{-1}\left(S_{1}\right)\left(\right.$ resp. $\left.K^{\times} \cdot \xi_{z}^{-1}(S)\right)$ of $K_{\mathbb{A}}^{\times}$.

Proof. As for the cases of $S$ and $S_{1}$, by Lemma 5 and [19], we have $k_{S}=k_{S_{1}}=\mathbb{Q}\left(\zeta_{N}\right), \Gamma_{S}=\mathbb{Q}^{\times} \Gamma(N)$ and $\Gamma_{S_{1}}=\mathbb{Q}^{\times} \Gamma_{1}(N)$. Since $N\left(j_{1, N}\right)$ (resp. $N\left(j_{N}\right)$ ) gives a model of the curve $X_{1}(N)$ (resp. $\left.X(N)\right)$, the assertion follows from [19], Proposition 6.33, and the argument mentioned above.

In particular, when $N=12$ we would obtain 
Corollary 10. Notations being as in Theorem $7, K\left(i, \sqrt{3}, N\left(j_{1,12}(z)\right)\right)$ is the class field of $K$ corresponding to the subgroup $K^{\times} \cdot \xi_{z}^{-1}\left(\mathbb{Q}^{\times} U_{1}\right)$ where $U_{1}=\left\{x=\left(x_{p}\right) \in U \mid x_{p} \in U_{1,(p)}\right.$ for all finite $\left.p\right\}$ and $U_{1,(p)}=\left\{\left(\begin{array}{ll}a & b \\ c & d\end{array}\right) \in\right.$ $\left.\mathrm{GL}_{2}\left(\mathbb{Z}_{p}\right) \mid a \equiv d \equiv 1, c \equiv 0\left(\bmod 12 \mathbb{Z}_{p}\right)\right\}$.

Since $\left(\begin{array}{cc}N & 0 \\ 0 & 1\end{array}\right)^{-1} \Gamma^{1}(N)\left(\begin{array}{cc}N & 0 \\ 0 & 1\end{array}\right)=\Gamma_{1}(N)$, we have

$$
K^{\times} \cdot \xi_{z / N}^{-1}\left(\mathbb{Q}^{\times} U_{1}\right)=K^{\times} \cdot \xi_{z}^{-1}\left(\mathbb{Q}^{\times} U^{1}\right) .
$$

Therefore we get the following corollary for $\Gamma^{1}(N)$.

Corollary 11. Notations being as in Theorem $7, N\left(j_{1, N}(z / N)\right)$ belongs to the maximal abelian extension $K^{\mathrm{ab}}$ of $K$ and $K\left(N\left(j_{1, N}(z / N)\right), \zeta_{N}\right)$ is the class field of $K$ corresponding to the subgroup $K^{\times} \cdot \xi_{z}^{-1}\left(S^{1}\right)$ of $K_{\mathbb{A}}^{\times}$.

4. Generation II. In view of standard results on complex multiplication, we are interested in investigating whether the value $j_{1,12}(\alpha)$ is a generator for a certain full ray class field when $\alpha$ is the quotient of a basis of an ideal belonging to the maximal order in an imaginary quadratic field. To this end we are first in need of a result from complex multiplication.

THEOREM 12. Let $\mathfrak{F}_{N}$ be the field of modular functions of level $N$ rational over $\mathbb{Q}\left(e^{2 \pi i / N}\right)$, and let $K$ be an imaginary quadratic field. Let $\mathcal{O}_{K}$ be the maximal order of $K$ and $\mathfrak{a}$ be an $\mathcal{O}_{K}$-ideal such that $\mathfrak{a}=\left[z_{1}, z_{2}\right]$ and $\alpha=$ $z_{1} / z_{2} \in \mathfrak{H}$. Then the field $K \mathfrak{F}_{N}(\alpha)$ generated over $K$ by all values $f(\alpha)$ with $f \in \mathfrak{F}_{N}$ and $f$ defined at $\alpha$, is the ray class field over $K$ with conductor $N$.

Proof. [16], Ch. 10, Corollary of Theorem 2.

Let $K\left(X\left(\Gamma^{\prime}\right)\right)$ be the function field of the modular curve $X\left(\Gamma^{\prime}\right)=\Gamma^{\prime} \backslash \mathfrak{H}^{*}$. Suppose that the genus of $X\left(\Gamma^{\prime}\right)$ is zero. Let $h$ be the width of the cusp $\infty$. By $F$ we denote the field of all modular functions in $K\left(X\left(\Gamma^{\prime}\right)\right)$ whose Fourier coefficients with respect to $q_{h}$ belong to $\mathbb{Q}$.

Lemma 13. Let $K\left(X\left(\Gamma^{\prime}\right)\right)=\mathbb{C}\left(J^{\prime}\right)$ for some $J^{\prime} \in K\left(X\left(\Gamma^{\prime}\right)\right)$. If $J^{\prime} \in F$, then $F=\mathbb{Q}\left(J^{\prime}\right)$.

Proof. [6], Lemma 4.

THEOREM $14 . \mathbb{Q}\left(j_{1,12}\right)$ is the the field of all modular functions in the field $K\left(X_{1}(12)\right)$ whose Fourier coefficients with respect to $q$ are rational numbers.

Proof. Since $j_{1,12}$ has rational Fourier coefficients, the result follows from Lemma 13.

It follows from [19], Proposition 6.9, that

$$
\mathfrak{F}_{N}=\mathbb{Q}\left(j, f_{\left(a_{1}, a_{2}\right)} \mid\left(a_{1}, a_{2}\right) \in N^{-1} \mathbb{Z}^{2}, \notin \mathbb{Z}^{2}\right) .
$$


Here $j$ is the classical modular function of level 1 and $f_{\left(a_{1}, a_{2}\right)}$ is the Fricke function defined by

$$
f_{a}(z)=\frac{g_{2}\left(\omega_{1}, \omega_{2}\right) g_{3}\left(\omega_{1}, \omega_{2}\right)}{\Delta\left(\omega_{1}, \omega_{2}\right)} \wp\left(a\left[\begin{array}{l}
\omega_{1} \\
\omega_{2}
\end{array}\right] ; \omega_{1}, \omega_{2}\right)
$$

for $z=\omega_{1} / \omega_{2} \in \mathfrak{H}$ and $a=\left(a_{1}, a_{2}\right)$. We recall that

(3) $f_{\left(a_{1}, a_{2}\right)}=f_{\left(b_{1}, b_{2}\right)}$ if and only if $\quad \pm\left(a_{1}, a_{2}\right) \equiv\left(b_{1}, b_{2}\right)\left(\bmod \mathbb{Z}^{2}\right)$

and

$$
\left.f_{\left(a_{1}, a_{2}\right)}\right|_{\gamma}=f_{\left(a_{1}, a_{2}\right) \gamma} \quad \text { for } \gamma \in \Gamma(1),
$$

where $\left.f(z)\right|_{\gamma}=f(\gamma z)$ for a modular function $f$.

Theorem 15. $K\left(X_{1}(12)\right)=\mathbb{C}\left(j, f_{(0, t)} \mid t \in 12^{-1} \mathbb{Z} \backslash \mathbb{Z}\right)\left(=\mathbb{C}\left(j_{1,12}\right)\right)$.

Proof. Observe that

$$
K(X(1)) \subseteq K\left(X_{1}(12)\right) \subseteq K(X(12))
$$

where $K(X(12))$ is a Galois extension over $K(X(1))$ with Galois group $\bar{\Gamma}(1) / \bar{\Gamma}(12)([18]$, Ch. VI, Theorem 4 or [19], p. 31). We consider the Galois group

$$
G=\operatorname{Gal}\left(K(X(12)) / \mathbb{C}\left(j, f_{(0, t)} \mid t \in 12^{-1} \mathbb{Z} \backslash \mathbb{Z}\right)\right) .
$$

For $\bar{\gamma} \in \bar{\Gamma}(1) / \bar{\Gamma}(12)$, let $\gamma=\left(\begin{array}{ll}a & b \\ c & d\end{array}\right)$ be its representative in $\Gamma(1)$. Then by (3) and (4),

$$
\begin{aligned}
\bar{\gamma} \in G & \Leftrightarrow f_{(0, t)}=\left.f_{(0, t)}\right|_{\gamma}=f_{(0, t) \gamma}=f_{(t c, t d)} \text { for } t \in 12^{-1} \mathbb{Z} \backslash \mathbb{Z} \\
& \Leftrightarrow(c, d) \equiv \pm(0,1)(\bmod 12) \\
& \Leftrightarrow \bar{\gamma} \in \bar{\Gamma}_{1}(12) .
\end{aligned}
$$

Hence we must have

$$
G=\bar{\Gamma}_{1}(12) / \bar{\Gamma}(12)=\operatorname{Gal}\left(K(X(12)) / K\left(X_{1}(12)\right)\right),
$$

from which we end up with $K\left(X_{1}(12)\right)=\mathbb{C}\left(j, f_{(0, t)} \mid t \in 12^{-1} \mathbb{Z} \backslash \mathbb{Z}\right)$.

Lemma 16. For $z \in \mathfrak{H}$, we get

$$
\mathbb{Q}\left(j(z), f_{(0, t)}(z) \mid t \in 12^{-1} \mathbb{Z} \backslash \mathbb{Z}\right)=\mathbb{Q}\left(j_{1,12}(z) / \sqrt{3}\right) .
$$

Proof. For $f \in K\left(X_{1}(12)\right)$, we let $W_{12}(f)=\left.f\right|_{\left(\begin{array}{cc}0 & -1 \\ 12 & 0\end{array}\right)}$ be the action of the Fricke involution. Since $W_{12}=\left(\begin{array}{cc}0 & -1 \\ 12 & 0\end{array}\right)$ belongs to the normalizer of $\Gamma_{1}(12)$ ([13]), $W_{12} \in \operatorname{Aut}\left(K\left(X_{1}(12)\right)\right)$. We observe that

$$
W_{12}(f)=\left.f\right|_{S}(12 z) \quad \text { for } S=\left(\begin{array}{cc}
0 & -1 \\
1 & 0
\end{array}\right) .
$$


Hence it follows that $W_{12}(j(z))=j(12 z)$ and $W_{12}\left(f_{(0, t)}(z)\right)=f_{(t, 0)}(12 z)$. Since $j_{1,12}(z)=\theta_{3}(2 z) / \theta_{3}(6 z)$, we derive, by (1),

$$
\begin{aligned}
\left.j_{1,12}(z)\right|_{S} & =\left.\frac{\theta_{3}(2 z)}{\theta_{3}(6 z)}\right|_{S}=\frac{\theta_{3}\left(-\frac{1}{z / 2}\right)}{\theta_{3}\left(-\frac{1}{z / 6}\right)} \\
& =\frac{\left(-i \frac{z}{2}\right)^{1 / 2} \theta_{3}\left(\frac{z}{2}\right)}{\left(-i \frac{z}{6}\right)^{1 / 2} \theta_{3}\left(\frac{z}{6}\right)}=\sqrt{3} / j_{1,12}\left(\frac{z}{12}\right) .
\end{aligned}
$$

We denote by $F_{1,12}$ the field of modular functions in $K\left(X_{1}(12)\right)$ with rational Fourier coefficients. Considering the Fourier expansions of Fricke functions ([16], p. 66, or [19], p. 141), we know that $f_{(t, 0)}(12 z)$ has rational Fourier coefficients for $t \in 12^{-1} \mathbb{Z} \backslash \mathbb{Z}$. Thus

$$
\mathbb{Q}\left(W_{12}(j(z)), W_{12}\left(f_{(0, t)}(z)\right) \mid t \in 12^{-1} \mathbb{Z} \backslash \mathbb{Z}\right) \subseteq F_{1,12} .
$$

Moreover, we observe by Theorem 15 that

$$
\begin{aligned}
\mathbb{C}\left(W_{12}(j(z)), W_{12}\left(f_{(0, t)}(z)\right) \mid t \in 12^{-1} \mathbb{Z} \backslash \mathbb{Z}\right) & =W_{12}\left(K\left(X_{1}(12)\right)\right) \\
& =K\left(X_{1}(12)\right) .
\end{aligned}
$$

On the other hand, by a similar argument to [6], Lemma 5, we get

$$
F_{1,12}=\mathbb{Q}\left(W_{12}(j(z)), W_{12}\left(f_{(0, t)}(z)\right) \mid t \in 12^{-1} \mathbb{Z} \backslash \mathbb{Z}\right) .
$$

We then deduce by Theorem 14 and (5) that

$$
F_{1,12}=\mathbb{Q}\left(j_{1,12}(z)\right)=\mathbb{Q}\left(W_{12}\left(j_{1,12}(z) / \sqrt{3}\right)\right),
$$

which by (6) forces

$$
W_{12}\left(\mathbb{Q}\left(j(z), f_{(0, t)}(z) \mid t \in 12^{-1} \mathbb{Z} \backslash \mathbb{Z}\right)\right)=W_{12}\left(\mathbb{Q}\left(j_{1,12}(z) / \sqrt{3}\right)\right) .
$$

Therefore applying the involution $W_{12}$ to the above yields the conclusion.

LEMma 17. We have

$$
\left\{\left(a_{1}, a_{2}\right)\left(\bmod \mathbb{Z}^{2}\right) \mid\left(a_{1}, a_{2}\right) \in 12^{-1} \mathbb{Z}^{2}, \notin \mathbb{Z}^{2}\right\}=A \cup B \cup C
$$

where

$$
\begin{aligned}
& A=\left\{\left(0, a_{1}\right)\left(\begin{array}{cc}
0 & -1 \\
1 & x
\end{array}\right)\left(\bmod \mathbb{Z}^{2}\right) \mid a_{1} \in 12^{-1} \mathbb{Z} \backslash \mathbb{Z}, x=0, \ldots, 11\right\}, \\
& B=\left\{\left(0, a_{2}\right)\left(\begin{array}{cc}
1 & 0 \\
x & 1
\end{array}\right)\left(\bmod \mathbb{Z}^{2}\right) \mid a_{2} \in 12^{-1} \mathbb{Z} \backslash \mathbb{Z}, x=0, \ldots, 11\right\}, \\
& C=\left\{\left(0, a_{2}\right)\left(\begin{array}{cc}
1 & 0 \\
y & 1
\end{array}\right)\left(\begin{array}{cc}
1 & -1 \\
0 & 1
\end{array}\right)\left(\bmod \mathbb{Z}^{2}\right) \mid a_{2} \in 12^{-1} \mathbb{Z} \backslash \mathbb{Z}, y=3,4,9,10\right\} .
\end{aligned}
$$

$\mathrm{Pr}$ o of. In order to generate the ray class field of an imaginary quadratic field $K$ with conductor 12 , we shall use Lemma 16 and the fact that

$$
\mathfrak{F}_{12}=\mathbb{Q}\left(j, f_{\left(a_{1}, a_{2}\right)} \mid\left(a_{1}, a_{2}\right) \in 12^{-1} \mathbb{Z}^{2}, \notin \mathbb{Z}^{2}\right) .
$$


To this end, considering lattice points (modulo 12) in a plane, divide the set proposed in the lemma into subsets by considering elements of the form $(0, t) \gamma$ with $\gamma \in \mathrm{SL}_{2}(\mathbb{Z})$. Observe that

$$
\begin{aligned}
& A=\left\{\left(a_{1}, a_{1} x\right) \mid a_{1} \in 12^{-1} \mathbb{Z} \backslash \mathbb{Z}, x=0, \ldots, 11\right\}, \\
& B=\left\{\left(a_{2} x, a_{2}\right) \mid a_{2} \in 12^{-1} \mathbb{Z} \backslash \mathbb{Z}, x=0, \ldots, 11\right\} .
\end{aligned}
$$

Direct computation shows that the elements not in $A \cup B$ form a set

$$
\begin{aligned}
E=\{(2,3),(2,9),(3,2),(3,4),(3,8),(3,10),(4,3),(4,6),(4,9),(6,4),(6,8), & \\
& (8,3),(8,6),(8,9),(9,2),(9,4),(9,8),(9,10),(10,3),(10,9)\} .
\end{aligned}
$$

Now we embed $E$ into a subset whose elements are of the form $(0, t) \gamma$ with $\gamma \in \mathrm{SL}_{2}(\mathbb{Z})$. Since $\left.\left(a_{1}, a_{2}\right)\right|_{T}=\left(a_{1}, a_{1}+a_{2}\right)(\bmod 12)$ for $T=\left(\begin{array}{ll}1 & 1 \\ 0 & 1\end{array}\right)$,

$$
\begin{aligned}
\left.E\right|_{T}=\{ & (2,5),(2,11),(3,5),(3,7),(3,11),(3,1),(4,7),(4,10),(4,1),(6,10), \\
& (6,2),(8,11),(8,2),(8,5),(9,11),(9,1),(9,5),(9,7),(10,1),(10,7)\} .
\end{aligned}
$$

It follows that the congruence $t^{\prime} y \equiv s^{\prime}(\bmod 12)$ yields $y=3,4,9$ or 10 , when $(s, t) T=\left(s^{\prime}, t^{\prime}\right)$ for $(s, t) \in E$. Thus we get

$$
\left.E\right|_{T} \subset\left\{\left(a_{2} y, a_{2}\right) \mid a_{2} \in 12^{-1} \mathbb{Z} \backslash \mathbb{Z}, y=3,4,9,10\right\} ;
$$

in other words,

$$
E \subset C=\left\{\left(0, a_{2}\right)\left(\begin{array}{ll}
1 & 0 \\
y & 1
\end{array}\right)\left(\begin{array}{cc}
1 & -1 \\
0 & 1
\end{array}\right) \mid a_{2} \in 12^{-1} \mathbb{Z} \backslash \mathbb{Z}, y=3,4,9,10\right\}
$$

which completes the proof.

Theorem 18. Let $K$ and $\alpha$ be as in Theorem 12 , and let $K_{(12)}$ denote the ray class field over $K$ with conductor 12 . Then

$$
\begin{aligned}
K_{(12)}=K & \left(j_{1,12}\left(\frac{-1}{\alpha+x}\right) / \sqrt{3}, j_{1,12}\left(\frac{\alpha}{x \alpha+1}\right) / \sqrt{3},\right. \\
& \left.j_{1,12}\left(\frac{\alpha-1}{y \alpha+1-y}\right) / \sqrt{3} \mid x=0, \ldots, 11 \text { and } y=3,4,9,10\right) .
\end{aligned}
$$

Proof. For each $z \in \mathfrak{H}$, we have

$$
\begin{aligned}
& \mathfrak{F}_{12}=\mathbb{Q}\left(j(z), f_{\left(a_{1}, a_{2}\right)}(z) \mid\left(a_{1}, a_{2}\right) \in 12^{-1} \mathbb{Z}^{2}, \notin \mathbb{Z}^{2}\right) \quad \text { by }(2) \\
& =\mathbb{Q}\left(j(z),\left.f_{\left(0, a_{1}\right)}\right|_{\left(\begin{array}{cc}
0 & -1 \\
1 & x
\end{array}\right)} \mid a_{1} \in 12^{-1} \mathbb{Z}, \notin \mathbb{Z}, x=0, \ldots, 11\right) \\
& \cup \mathbb{Q}\left(j(z),\left.f_{\left(0, a_{2}\right)}\right|_{\left(\begin{array}{ll}
1 & 0 \\
x & 1
\end{array}\right)} \mid a_{2} \in 12^{-1} \mathbb{Z}, \notin \mathbb{Z}, x=0, \ldots, 11\right) \\
& \cup \mathbb{Q}\left(j(z), f_{\left(0, a_{2}\right)}\left|\left(\begin{array}{cc}
1 & 0 \\
y & 1
\end{array}\right)\left(\begin{array}{cc}
1 & -1 \\
0 & 1
\end{array}\right)\right| a_{2} \in 12^{-1} \mathbb{Z}, \notin \mathbb{Z}, y=3,4,9,10\right)
\end{aligned}
$$




$$
\begin{array}{r}
=\mathbb{Q}\left(j_{1,12}\left(\frac{-1}{z+x}\right) / \sqrt{3}, j_{1,12}\left(\frac{z}{x z+1}\right) / \sqrt{3}, j_{1,12}\left(\frac{z-1}{y z+1-y}\right) / \sqrt{3}\right. \\
\mid x=0, \ldots, 11 \text { and } y=3,4,9,10) \quad \text { by Lemma } 16 .
\end{array}
$$

Therefore, the result follows from Theorem 12 .

By class field theory ([19], Section 5.2, or [21], Theorem 3.6), the reciprocity map induces an isomorphism

$$
[\cdot, K]: K_{\mathbb{A}}^{\times} / K^{\times} U_{(12)} \stackrel{\sim}{\rightarrow} \operatorname{Gal}\left(K_{(12)} / K\right)
$$

where $U_{(12)}$ is the subgroup of $K_{\mathbb{A}}^{\times}$given by

$$
\begin{array}{r}
U_{(12)}=\left\{s \in K_{\mathbb{A}}^{\times} \mid s_{\mathfrak{p}} \in \mathcal{O}_{\mathfrak{p}}^{\times} \text {and } s_{\mathfrak{p}} \equiv 1\left(\bmod (12) \mathcal{O}_{\mathfrak{p}}\right)\right. \\
\text { for all finite primes } \mathfrak{p}\} .
\end{array}
$$

5. Generation III. Let $K$ be an imaginary quadratic field, $\mathcal{O}_{K}$ the maximal order of $K$ and $\mathfrak{a}=\left[z_{1}, z_{2}\right]$ an $\mathcal{O}_{K}$-ideal with $\alpha:=z_{1} / z_{2} \in \mathfrak{H}$. Since $\alpha$ is an imaginary quadratic element, $\alpha$ satisfies an integral equation $a z^{2}+b z+c=0$. In this section, we shall find class fields generated by singular values $j_{1,12}(\alpha)$ and $j_{1,12}(\alpha)^{2}$ under some conditions on $a$ and the discriminant $d_{K}\left(=b^{2}-4 a c\right)$ of $K$. First, we need the following lemma which is a modification of a statement in the proof of Theorem 3.7.5 in [1].

LEMma 19. Let $f$ be a modular function of level 12 with rational Fourier coefficients and $(\beta)$ a principal ideal of $\mathcal{O}_{K}$ relatively prime to 12 . Put $\beta=m+n(a \alpha) \in \mathbb{Z}+\mathbb{Z}(a \alpha)=\mathcal{O}_{K}$ and let $\mathcal{A}_{\beta}$ be a matrix in $\mathrm{SL}_{2}(\mathbb{Z})$ whose image in $\mathrm{SL}_{2}(\mathbb{Z} / 12 \mathbb{Z})$ is equal to

$$
\left(\begin{array}{cc}
-b n+m & -c n \\
a n N(\beta)^{-1} & m N(\beta)^{-1}
\end{array}\right) .
$$

Then the action of $(\beta)$ on $f(\alpha)$ is given by

$$
f(\alpha)^{\left[(\beta), K_{(12)} / K\right]}=f\left(\mathcal{A}_{\beta} \cdot \alpha\right) .
$$

In Theorem 18, we generated the ray class field $K_{(12)}$ over $K$ by 28 singular values of $j_{1,12}$. However, whenever $a$ is relatively prime to 12 , we now see that $K_{(12)}$ is simply generated by one singular value $j_{1,12}(\alpha)$ and, moreover, $j_{0,12}(\alpha)$ defined below spans some ring class field.

Theorem 20. Notations being as above, let $a z^{2}+b z+c=0$ be the equation of $\alpha$ such that $a>0,(a, b, c)=1$, and let $j_{0,12}(z)=j_{1,12}(z)^{2}=$ $\theta_{3}(2 z)^{2} / \theta_{3}(6 z)^{2}$. Suppose that $(a, 12)=1$. Then:

(1) $j_{0,12}(\alpha)$ generates the ring class field of an imaginary quadratic order $\mathcal{O}\left(=\mathbb{Z}+12 \mathcal{O}_{K}\right)$ with discriminant $12^{2} d_{K}$. 
(2) $j_{1,12}(\alpha)$ generates the ray class field $K_{(12)}$ of $K$ with conductor 12 , and the degree of $K\left(j_{1,12}(\alpha)\right)$ over $K$ is $2 h(\mathcal{O})$, where $h(\mathcal{O})$ is the class number of $\mathcal{O}$.

Proof. (1) By Theorem 1(1), $j_{0,12}(z) \in K\left(X_{0}(12)\right)$. We observe that

$$
\left[K\left(X_{1}(12)\right): \mathbb{C}\left(j_{0,12}(z)\right)\right]=\left[\mathbb{C}\left(j_{1,12}(z)\right): \mathbb{C}\left(j_{0,12}(z)\right)\right]=2 .
$$

Since $\left[\bar{\Gamma}_{0}(N): \bar{\Gamma}_{1}(N)\right]=\frac{1}{2} \phi(N)$ for $N>2$, with $\phi$ the Euler phi function, it follows that $\left[K\left(X_{1}(12)\right): K\left(X_{0}(12)\right)\right]=\left[\bar{\Gamma}_{0}(12): \bar{\Gamma}_{1}(12)\right]=2$; whence $K\left(X_{0}(12)\right)=\mathbb{C}\left(j_{0,12}(z)\right)$. This indicates that $j_{0,12}(z)$ is a field generator of a genus zero curve, and so we are able to normalize it as

$$
N\left(j_{0,12}(z)\right)=\frac{4}{j_{0,12}(z)-1}+1=T_{12 I}(z),
$$

the Thompson series of type 12I. Now the result follows from [1], Theorem 3.7.5(1).

(2) Let $L_{0}=K\left(j_{0,12}(\alpha)\right)$ and $L_{1}=K\left(j_{1,12}(\alpha)\right)$. Then we have the following field tower:

$$
K \subseteq L_{0} \subseteq L_{1} \subseteq K_{(12)}
$$

Here the last inclusion follows from Theorem 12. For a subfield $L$ of $K_{(12)}$, let $\Phi_{L / K}: I_{K}(12) \rightarrow \operatorname{Gal}(L / K)$ signify the Artin map, where $I_{K}(12)=$ fractional ideal $\left.\mathfrak{a} \mid\left(\mathfrak{a}, 12 \mathcal{O}_{K}\right)=1\right\}$, which forms a group under multiplication. Then $\operatorname{Ker}\left(\Phi_{K_{(12)} / K}\right)=P_{K, 1}(12)$ and

$$
P_{K, 1}(12) \subseteq \operatorname{Ker}\left(\Phi_{L_{1} / K}\right) \subseteq \operatorname{Ker}\left(\Phi_{L_{0} / K}\right) \subseteq I_{K}(12)
$$

by class field theory, where $P_{K, 1}(12)$ denotes the subgroup of $I_{K}(12)$ generated by the principal ideals $\beta \mathcal{O}_{K}$ with $\beta \in \mathcal{O}_{K}$ and $\beta \equiv 1\left(\bmod 12 \mathcal{O}_{K}\right)$. Since $L_{0}$ is the ring class field of $\mathcal{O}=\mathbb{Z}+12 \mathcal{O}_{K}$, it follows from class field theory (e.g. [3]) that

$$
\operatorname{Pic}(\mathcal{O})=I(\mathcal{O}, 12) / P(\mathcal{O}, 12) \cong I_{K}(12) / P_{K, \mathbb{Z}}(12) \cong \operatorname{Gal}\left(L_{0} / K\right),
$$

where the last isomorphism is induced by the Artin map $\Phi_{L_{0} / K}$, and $P_{K, \mathbb{Z}}(12)$ denotes the subgroup of $I_{K}(12)$ generated by the principal ideals $\beta \mathcal{O}_{K}$ with $\beta \in \mathcal{O}_{K}$ and $\beta \equiv l\left(\bmod 12 \mathcal{O}_{K}\right)$ for some integer $l$ relatively prime to 12 . Therefore we get $\operatorname{Ker}\left(\Phi_{L_{0} / K}\right)=P_{K, \mathbb{Z}}(12)$ and

$$
P_{K, 1}(12) \subseteq \operatorname{Ker}\left(\Phi_{L_{1} / K}\right) \subseteq P_{K, \mathbb{Z}}(12) .
$$

Since $P_{K, \mathbb{Z}}(12) / P_{K, 1}(12)$ is isomorphic to $(\mathbb{Z} / 12 \mathbb{Z})^{\times} /\{ \pm 1\}$, the degree of $P_{K, \mathbb{Z}}(12)$ over $P_{K, 1}(12)$ is 2 . Thus we have either $\operatorname{Ker}\left(\Phi_{L_{1} / K}\right)=P_{K, 1}(12)$ or $\operatorname{Ker}\left(\Phi_{L_{1} / K}\right)=P_{K, \mathbb{Z}}(12)$, and hence it remains to prove $\operatorname{Ker}\left(\Phi_{L_{1} / K}\right)=$ $P_{K, 1}(12)$.

Now, we take two integers $n$ and $m$ such that $12 \mid n$ and $m \equiv \pm 5$ $(\bmod 12)$. Let $(\beta)$ be a principal ideal of $\mathcal{O}_{K}$ prime to 12 , and $\mathcal{A}_{\beta}$ be 
as in Lemma 19. Then $\mathcal{A}_{\beta} \in \Gamma_{0}(12) \backslash \pm \Gamma_{1}(12)$, and since

$$
\chi_{3}\left(m \cdot N(\beta)^{-1}\right)=\left(\frac{3}{m}\right)\left(\frac{1}{N(\beta)^{-1}}\right)=-1 \cdot 1=-1,
$$

we get $j_{1,12}\left(\mathcal{A}_{\beta} \cdot \alpha\right)=-j_{1,12}(\alpha)$ by Theorem $1(1)$. Since $j_{1,12}$ never vanishes on $\mathfrak{H}$, we must have $j_{1,12}\left(\mathcal{A}_{\beta} \cdot \alpha\right) \neq j_{1,12}(\alpha)$.

On the other hand, $j_{0,12}\left(\mathcal{A}_{\beta} \cdot \alpha\right)=j_{1,12}\left(\mathcal{A}_{\beta} \cdot \alpha\right)^{2}=j_{0,12}(\alpha)$, from which we get $(\beta) \in \operatorname{Ker}\left(\Phi_{L_{0} / K}\right) \backslash \operatorname{Ker}\left(\Phi_{L_{1} / K}\right)$. Therefore $\operatorname{Ker}\left(\Phi_{L_{1} / K}\right)$ is equal to $P_{K, 1}(12)$, and $L_{1}=K_{(12)}$ by class field theory. The last assertion follows from the fact that $j_{0,12}(\alpha)$ generates the ring class field of $\mathcal{O}$ and $\left[K\left(j_{1,12}(\alpha)\right): K\left(j_{0,12}(\alpha)\right)\right]=2$.

ExAmples. Put $K=\mathbb{Q}(\sqrt{N})$ with $N$ a square-free negative integer. Then $j_{0,12}((1+\sqrt{N}) / 2)$ (resp. $j_{0,12}(\sqrt{N})$ ) generates the ring class field of an imaginary quadratic order $\mathcal{O}\left(=\mathbb{Z}+12 \mathcal{O}_{K}\right)$ with discriminant $12^{2} d_{K}$ provided that $N \equiv 1(\bmod 4)($ resp. $N \equiv 2,3(\bmod 4))$ and $j_{1,12}((1+$ $\sqrt{N}) / 2$ ) (resp. $j_{1,12}(\sqrt{N})$ ) generates the ray class field $K_{(12)}$ of $K$ with conductor 12 if $N \equiv 1(\bmod 4)($ resp. $N \equiv 2,3(\bmod 4))$.

As for the construction of the ray class fields over imaginary quadratic fields with conductor strictly dividing 12 , we need to consider some other conditions on $a$ and $d_{K}$, different from the previous one. We shall illustrate this in two theorems; one excluding the cases $d_{K}=-3$ and -4 , the other only with $d_{K}=-3$ and -4 .

Theorem 21. Notations being as above, let $a z^{2}+b z+c=0$ be the equation of $\alpha$ such that $a>0$ and $(a, b, c)=1$, and let $K_{\mathfrak{f}}$ be a ray class field over $K$ with conductor $\mathfrak{f}$. Assume that the discriminant of $K$ is neither -4 nor -3 (i.e. $K \neq \mathbb{Q}(\sqrt{-1}), \mathbb{Q}(\sqrt{-3}))$. Then:

(1) If $(a, 12)=2$, then $j_{1,12}(\alpha)$ generates $K_{\mathfrak{f}}$ over $K$ with conductor $\mathfrak{f}$ given by

$$
\mathfrak{f}=\left\{\begin{array}{lll}
3[2, a \alpha]^{3}, & d_{K} \equiv 0(\bmod 4), \\
3[2, a \alpha][2, a \alpha+1]^{2}, & d_{K} \equiv 1(\bmod 8) .
\end{array}\right.
$$

Furthermore, 2 ramifies in $K$ when $d_{K} \equiv 0(\bmod 4)$ and splits completely in $K$ if $d_{K} \equiv 1(\bmod 8)$, and so

$$
12 \mathcal{O}_{K}= \begin{cases}3[2, a \alpha]^{4}, & d_{K} \equiv 0(\bmod 4), \\ 3[2, a \alpha]^{2}[2, a \alpha+1]^{2}, & d_{K} \equiv 1(\bmod 8) .\end{cases}
$$

(2) If $(a, 12)=3$, then $j_{1,12}(\alpha)$ generates $K_{\mathfrak{f}}$ with conductor $\mathfrak{f}$ given by

$$
\mathfrak{f}= \begin{cases}4[3, a \alpha], & b \equiv 0(\bmod 3), \\ 4[3, a \alpha+1], & b \equiv 1(\bmod 3), \\ 4[3, a \alpha+2], & b \equiv 2(\bmod 3)\end{cases}
$$


Moreover,

$$
12 \mathcal{O}_{K}= \begin{cases}4[3, a \alpha]^{2}, & b \equiv 0(\bmod 3), \\ 4[3, a \alpha][3, a \alpha+1], & b \equiv 1(\bmod 3), \\ 4[3, a \alpha][3, a \alpha+2], & b \equiv 2(\bmod 3) .\end{cases}
$$

(3) If $(a, 12)=4$ and $d_{K} \equiv 1(\bmod 8)$, then $j_{1,12}(\alpha)$ generates $K_{\mathfrak{f}}$ with conductor $\mathfrak{f}=3[2, a \alpha+1]^{2}$ and $12 \mathcal{O}_{K}=3[2, a \alpha]^{2}[2, a \alpha+1]^{2}$.

(4) If $(a, 12)=6$ and $d_{K} \not \equiv 5(\bmod 8)$, then $j_{1,12}(\alpha)$ generates $K_{\mathfrak{f}}$ with conductor $\mathfrak{f}$ given by

$$
\mathfrak{f}= \begin{cases}{[2, a \alpha]^{3}[3, a \alpha],} & b \equiv 0(\bmod 6) \\ {[2, a \alpha][2, a \alpha+1]^{2}[3, a \alpha+1],} & b \equiv 1(\bmod 6) \\ {[2, a \alpha]^{3}[3, a \alpha+2],} & b \equiv 2(\bmod 6) \\ {[2, a \alpha][2, a \alpha+1]^{2}[3, a \alpha],} & b \equiv 3(\bmod 6) \\ {[2, a \alpha]^{3}[3, a \alpha+1],} & b \equiv 4(\bmod 6) \\ {[2, a \alpha][2, a \alpha+1]^{2}[3, a \alpha+2],} & b \equiv 5(\bmod 6)\end{cases}
$$

Moreover,

$$
12 \mathcal{O}_{K}= \begin{cases}{[2, a \alpha]^{4}[3, a \alpha]^{2},} & b \equiv 0(\bmod 6), \\ {[2, a \alpha]^{2}[2, a \alpha+1]^{2}[3, a \alpha][3, a \alpha+1],} & b \equiv 1(\bmod 6), \\ {[2, a \alpha]^{4}[3, a \alpha][3, a \alpha+2],} & b \equiv 2(\bmod 6), \\ {[2, a \alpha]^{2}[2, a \alpha+1]^{2}[3, a \alpha]^{2},} & b \equiv 3(\bmod 6), \\ {[2, a \alpha]^{4}[3, a \alpha][3, a \alpha+1],} & b \equiv 4(\bmod 6), \\ {[2, a \alpha]^{2}[2, a \alpha+1]^{2}[3, a \alpha][3, a \alpha+2],} & b \equiv 5(\bmod 6) .\end{cases}
$$

(5) If $(a, 12)=12$ and $d_{K} \equiv 1(\bmod 8)$, then $j_{1,12}(\alpha)$ generates $K_{\mathfrak{f}}$ with conductor $\mathfrak{f}$ given by

$$
\mathfrak{f}= \begin{cases}{[2, a \alpha+1]^{2}[3, a \alpha],} & b \equiv 0(\bmod 3), \\ {[2, a \alpha+1]^{2}[3, a \alpha+1],} & b \equiv 1(\bmod 3), \\ {[2, a \alpha+1]^{2}[3, a \alpha+2],} & b \equiv 2(\bmod 3) .\end{cases}
$$

Further,

$$
12 \mathcal{O}_{K}= \begin{cases}{[2, a \alpha]^{2}[2, a \alpha+1]^{2}[3, a \alpha]^{2},} & b \equiv 0(\bmod 3), \\ {[2, a \alpha]^{2}[2, a \alpha+1]^{2}[3, a \alpha][3, a \alpha+1],} & b \equiv 1(\bmod 3), \\ {[2, a \alpha]^{2}[2, a \alpha+1]^{2}[3, a \alpha][3, a \alpha+2],} & b \equiv 2(\bmod 3) .\end{cases}
$$

Pr o of. As in Theorem 20, for a subfield $L$ of $K_{(12)}$, let $\Phi_{L / K}: I_{K}(12) \rightarrow$ $\operatorname{Gal}(L / K)$ be the Artin map. Since $j_{1,12}(\alpha) \in K_{(12)}$ by Theorem 12, we have $K \subseteq K\left(j_{1,12}(\alpha)\right) \subseteq K_{(12)}$ so that

$$
P_{K, 1}(12)=\operatorname{Ker}\left(\Phi_{K_{(12)} / K}\right) \subseteq \operatorname{Ker}\left(\Phi_{K\left(j_{1,12}(\alpha)\right) / K}\right) .
$$

Let $\mathfrak{a} \in \operatorname{Ker}\left(\Phi_{K\left(j_{1,12}(\alpha)\right) / K}\right)$. Then $\Phi_{K\left(j_{1,12}(\alpha)\right) / K}(\mathfrak{a})=\left[\mathfrak{a}, K\left(j_{1,12}(\alpha)\right) / K\right]$ fixes $j_{1,12}(\alpha)$ and hence it fixes $j(\alpha)$, too. Since $K(j(\alpha))$ is the Hilbert class field of $K, I_{K} / P_{K} \cong \operatorname{Gal}(K(j(\alpha)) / K)$. And the fact that $\left[\mathfrak{a}, K\left(j_{1,12}(\alpha)\right) / K\right]$ is trivial on $K(j(\alpha))$ implies $\mathfrak{a} \in P_{K} \cap I_{K}(12)=P_{K}(12)$. 
Now we write $\mathfrak{a}=\beta \mathcal{O}_{K}$ with $\beta \in \mathcal{O}_{K}$ and $(N(\beta), 12)=1$. Let $\beta=$ $m+n(a \alpha) \in \mathbb{Z}+\mathbb{Z} \cdot(a \alpha)=\mathcal{O}_{K}$. Considering $\mathcal{A}_{\beta}$ described in Lemma 19, we see that $(\beta) \in \operatorname{Ker}\left(\Phi_{K\left(j_{1,12}(\alpha)\right) / K}\right)$ if and only if $\mathcal{A}_{\beta} \in \pm \Gamma_{1}(12) \cdot \Gamma_{\alpha}$, where $\Gamma_{\alpha}=\left\{\gamma \in \mathrm{SL}_{2}(\mathbb{Z}) \mid \gamma(\alpha)=\alpha\right\}$. Note that $\Gamma_{\alpha}$ is nontrivial if and only if $\alpha$ is equivalent to $i$ or $\varrho=e^{2 \pi i / 3}$ under the action of $\mathrm{SL}_{2}(\mathbb{Z})$. In view of quadratic forms we see that $\Gamma_{\alpha}$ is nontrivial if and only if $d_{K}=-4$ or -3 , that is, $K=\mathbb{Q}(\sqrt{-1})$ or $K=\mathbb{Q}(\sqrt{-3})$. By our assumption, however, $\Gamma_{\alpha}$ must be trivial; hence

$$
(\beta) \in \operatorname{Ker}\left(\Phi_{K\left(j_{1,12}(\alpha)\right) / K}\right) \Leftrightarrow \mathcal{A}_{\beta} \in \pm \Gamma_{1}(12) .
$$

(1) Suppose that $(a, 12)=2$. Then, for $(\beta) \in I_{K}(12)$,

$$
\begin{aligned}
(\beta) \in \operatorname{Ker}\left(\Phi_{K\left(j_{1,12}(\alpha)\right) / K}\right) & \Leftrightarrow \mathcal{A}_{\beta} \in \pm \Gamma_{1}(12) \\
& \Leftrightarrow 12 \mid \text { an and }-b n+m \equiv \pm 1 \quad(\bmod 12) \\
& \Leftrightarrow 6 \mid n \text { and } m \in \pm 1+b n+12 \mathbb{Z} \text { since }(a, 12)=2 \\
& \Leftrightarrow \pm \beta \in 1+6[2, a \alpha+b] \\
& \Leftrightarrow(\beta) \in P_{K, 1}(\mathfrak{f}) \text { with } \mathfrak{f}=6[2, a \alpha+b] .
\end{aligned}
$$

Therefore we have

$$
\operatorname{Gal}\left(K\left(j_{1,12}(\alpha)\right) / K\right) \cong I_{K}(12) / P_{K, 1}(\mathfrak{f}) \cap I_{K}(12) \cong I_{K}(\mathfrak{f}) / P_{K, 1}(\mathfrak{f}),
$$

and $K\left(j_{1,12}(\alpha)\right)=K_{\mathfrak{f}}$ by class field theory.

We observe that $[2, a \alpha+b]$ is the prime ideal $\mathfrak{p}$ of $K$ lying above $2 \mathbb{Z}$ which would be $[2, a \alpha](\operatorname{resp} .[2, a \alpha+1])$ if $d_{K} \equiv 0(\bmod 4)\left(\operatorname{resp} . d_{K} \equiv 1\right.$ $(\bmod 8)$ ). Since the polynomial $X^{2}+b X+a c$ of $a \alpha$ is congruent to

$$
\begin{cases}X^{2}(\bmod 2) & \text { if } d_{K} \equiv 0(\bmod 4) \\ X(X+1)(\bmod 2) & \text { if } d_{K} \equiv 1(\bmod 8)\end{cases}
$$

we see that 2 ramifies into $[2, a \alpha]^{2}$ when $d_{K} \equiv 0(\bmod 4)$ and splits completely into $[2, a \alpha][2, a \alpha+1]$ if $d_{K} \equiv 1(\bmod 8)$. Note that $I_{K}(12)=I_{K}(\mathfrak{f})$ because

$$
\mathfrak{f}(=6 \mathfrak{p})= \begin{cases}3[2, a \alpha]^{3}, & d_{K} \equiv 0(\bmod 4), \\ 3[2, a \alpha][2, a \alpha+1]^{2}, & d_{K} \equiv 1(\bmod 8)\end{cases}
$$

and

$$
12 \mathcal{O}_{K}= \begin{cases}3[2, a \alpha]^{4}, & d_{K} \equiv 0(\bmod 4), \\ 3[2, a \alpha]^{2}[2, a \alpha+1]^{2}, & d_{K} \equiv 1(\bmod 8) .\end{cases}
$$

(2) Assume that $(a, 12)=3$. Then, in a similar manner, we find that for $(\beta) \in I_{K}(12)$,

$$
\begin{aligned}
(\beta) \in \operatorname{Ker}\left(\Phi_{K\left(j_{1,12}(\alpha)\right) / K}\right) & \Leftrightarrow \mathcal{A}_{\beta} \in \pm \Gamma_{1}(12) \\
& \Leftrightarrow \pm \beta \in 1+4[3, a \alpha+b] \\
& \Leftrightarrow(\beta) \in P_{K, 1}(\mathfrak{f}) \text { with } \mathfrak{f}=4[3, a \alpha+b] .
\end{aligned}
$$


Hence $\operatorname{Ker}\left(\Phi_{K\left(j_{1,12}(\alpha)\right) / K}\right)=P_{K, 1}(\mathfrak{f}) \cap I_{K}(12)$, and so $K\left(j_{1,12}(\alpha)\right)=K_{\mathfrak{f}}$.

Here, we note that the prime ideal $[3, a \alpha+b]$ would be $[3, a \alpha+i]$ if $b \equiv i$ $(\bmod 3)$ for $i=0,1,2$. Since the polynomial $X^{2}+b X+a c$ of $a \alpha$ is congruent to

$$
\begin{cases}X^{2}(\bmod 3) & \text { if } b \equiv 0(\bmod 3) \\ X(X+1)(\bmod 3) & \text { if } b \equiv 1(\bmod 3) \\ X(X+2)(\bmod 3) & \text { if } b \equiv 2(\bmod 3)\end{cases}
$$

we claim that 3 ramifies into $[3, a \alpha]^{2}$ when $b \equiv 0(\bmod 3)$ and splits completely into $[3, a \alpha][3, a \alpha+1](\operatorname{resp} .[3, a \alpha][3, a \alpha+2])$ when $b \equiv 1(\bmod 3)$ $($ resp. $b \equiv 2(\bmod 3))$. Observe in addition that $I_{K}(12)=I_{K}(\mathfrak{f})$ only if $d_{K} \equiv 0(\bmod 3)($ i.e. $b \equiv 0(\bmod 3))$ because

$$
\begin{array}{ll}
\mathfrak{f}=4[3, a \alpha], & 12 \mathcal{O}_{K}=4[3, a \alpha]^{2}, \\
\mathfrak{f}=4[3, a \alpha+1], & 12 \mathcal{O}_{K}=4[3, a \alpha][3, a \alpha+1], \\
\mathfrak{f}=4[3, a \alpha+2], & 12 \mathcal{O}_{K}=4[3, a \alpha][3, a \alpha+2] .
\end{array}
$$

(3) Assume that $(a, 12)=4$. Then, for $(\beta) \in I_{K}(12)$,

$$
\begin{aligned}
(\beta) \in \operatorname{Ker}\left(\Phi_{K\left(j_{1,12}(\alpha)\right) / K}\right) & \Leftrightarrow \mathcal{A}_{\beta} \in \pm \Gamma_{1}(12) \\
& \Leftrightarrow 12 \mid \text { an and }-b n+m \equiv \pm 1(\bmod 12) \\
& \Leftrightarrow 3 \mid n \text { and } m \in \pm 1+b n+12 \mathbb{Z} \text { since }(a, 12)=4 \\
& \Leftrightarrow \pm \beta \in 1+3[4, a \alpha+b] .
\end{aligned}
$$

Due to $d_{K} \equiv 1(\bmod 8)$ one can easily show that $[4, a \alpha+b]=[2, a \alpha+1]^{2}$. Therefore, $K\left(j_{1,12}(\alpha)\right)=K_{\mathfrak{f}}$ with $\mathfrak{f}=3[2, a \alpha+1]^{2}$.

(4) Assume that $(a, 12)=6$. Then, for $(\beta) \in I_{K}(12)$,

$$
\begin{aligned}
(\beta) \in \operatorname{Ker}\left(\Phi_{K\left(j_{1,12}(\alpha)\right) / K}\right) & \\
& \Leftrightarrow \mathcal{A}_{\beta} \in \pm \Gamma_{1}(12) \\
& \Leftrightarrow \pm \beta \in 1+2[6, a \alpha+b]=1+2[2, a \alpha+b][3, a \alpha+b] \\
& \Leftrightarrow(\beta) \in P_{K, 1}(\mathfrak{f}) \text { with } \mathfrak{f}=2[2, a \alpha+b][3, a \alpha+b] .
\end{aligned}
$$

We conclude that $K\left(j_{1,12}(\alpha)\right)=K_{\mathfrak{f}}$. Note that $[6, a \alpha+b]$ is equal to

$[2, a \alpha][3, a \alpha], \quad b \equiv 0(\bmod 6), \quad[2, a \alpha+1][3, a \alpha+1], \quad b \equiv 1(\bmod 6)$,

$[2, a \alpha][3, a \alpha+2], \quad b \equiv 2(\bmod 6), \quad[2, a \alpha+1][3, a \alpha], \quad b \equiv 3(\bmod 6)$, $[2, a \alpha][3, a \alpha+1], \quad b \equiv 4(\bmod 6), \quad[2, a \alpha+1][3, a \alpha+2], \quad b \equiv 5(\bmod 6)$. 
Since the polynomial $X^{2}+b X+a c$ of $a \alpha$ is congruent to

$$
\begin{cases}X^{2}(\bmod 2), X^{2}(\bmod 3) & \text { if } b \equiv 0(\bmod 6), \\ X(X+1)(\bmod 2), X(X+1)(\bmod 3) & \text { if } b \equiv 1(\bmod 6), \\ X^{2}(\bmod 2), X(X+2)(\bmod 3) & \text { if } b \equiv 2(\bmod 6), \\ X(X+1)(\bmod 2), X^{2}(\bmod 3) & \text { if } b \equiv 3(\bmod 6), \\ X^{2}(\bmod 2), X(X+1)(\bmod 3) & \text { if } b \equiv 4(\bmod 6), \\ X(X+1)(\bmod 2), X(X+2)(\bmod 3) & \text { if } b \equiv 5(\bmod 6),\end{cases}
$$

we see that 2 (resp. 3) ramifies into $[2, a \alpha]^{2}$ (resp. $\left.[3, a \alpha]^{2}\right)$ when $d_{K} \equiv 0$ $(\bmod 6)($ i.e. $b \equiv 0(\bmod 6))$, and either 2 or 3 splits completely otherwise. Moreover, observe that $I_{K}(12)=I_{K}(\mathfrak{f})$ only if $b \equiv 0$ or $3(\bmod 6)$ because

- if $b \equiv 0(\bmod 6)$ then

$$
\mathfrak{f}=[2, a \alpha]^{3}[3, a \alpha], \quad 12 \mathcal{O}_{K}=[2, a \alpha]^{4}[3, a \alpha]^{2},
$$

- if $b \equiv 1(\bmod 6)$ then

$\mathfrak{f}=[2, a \alpha][2, a \alpha+1]^{2}[3, a \alpha+1], \quad 12 \mathcal{O}_{K}=[2, a \alpha]^{2}[2, a \alpha+1]^{2}[3, a \alpha][3, a \alpha+1]$,

- if $b \equiv 2(\bmod 6)$ then

$$
\mathfrak{f}=[2, a \alpha]^{3}[3, a \alpha+2], \quad 12 \mathcal{O}_{K}=[2, a \alpha]^{4}[3, a \alpha][3, a \alpha+2],
$$

- if $b \equiv 3(\bmod 6)$ then

$$
\mathfrak{f}=[2, a \alpha][2, a \alpha+1]^{2}[3, a \alpha], \quad 12 \mathcal{O}_{K}=[2, a \alpha]^{2}[2, a \alpha+1]^{2}[3, a \alpha]^{2},
$$

- if $b \equiv 4(\bmod 6)$ then

$$
\mathfrak{f}=[2, a \alpha]^{3}[3, a \alpha+1], \quad 12 \mathcal{O}_{K}=[2, a \alpha]^{4}[3, a \alpha][3, a \alpha+1],
$$

- if $b \equiv 5(\bmod 6)$ then

$\mathfrak{f}=[2, a \alpha][2, a \alpha+1]^{2}[3, a \alpha+2], \quad 12 \mathcal{O}_{K}=[2, a \alpha]^{2}[2, a \alpha+1]^{2}[3, a \alpha][3, a \alpha+2]$.

(5) Assume that $(a, 12)=12$. Then, for $(\beta) \in I_{K}(12)$,

$$
\begin{aligned}
(\beta) \in \operatorname{Ker}\left(\Phi_{K\left(j_{1,12}(\alpha)\right) / K}\right) & \Leftrightarrow \mathcal{A}_{\beta} \in \pm \Gamma_{1}(12) \\
& \Leftrightarrow 12 \mid \text { an and }-b n+m \equiv \pm 1(\bmod 12) \\
& \Leftrightarrow m \in \pm 1+b n+12 \mathbb{Z} \text { since }(a, 12)=12 \\
& \Leftrightarrow \pm \beta \in 1+[12, a \alpha+b]=1+[3, a \alpha+b][4, a \alpha+b] .
\end{aligned}
$$

Therefore $K\left(j_{1,12}(\alpha)\right)=K_{\mathfrak{f}}$ with $\mathfrak{f}=[3, a \alpha+b][4, a \alpha+b]$. Note that the conductor $\mathfrak{f}$ would be

$$
\begin{cases}{[2, a \alpha+1]^{2}[3, a \alpha],} & b \equiv 0(\bmod 3) \\ {[2, a \alpha+1]^{2}[3, a \alpha+1],} & b \equiv 1(\bmod 3) \\ {[2, a \alpha+1]^{2}[3, a \alpha+2],} & b \equiv 2(\bmod 3)\end{cases}
$$


Remark 22. (1) In the cases $(a, 12)=2,4,6$ and 12 , if $d_{K} \equiv 5(\bmod 8)$, there is no $\alpha$ satisfying the hypothesis.

(2) In the cases $(a, 12)=4$ and 12 , we see that $[4, a \alpha+b](=[4, a \alpha]$ or $[4, a \alpha+2])$ does not divide $2 \mathcal{O}_{K}$ if $d_{K} \equiv 0(\bmod 4)$.

Examples. (1) Take $K=\mathbb{Q}(\sqrt{-2})$ and $\mathfrak{a}=[2, \sqrt{-2}]$. Then $d_{K}=-8 \equiv$ $0(\bmod 4)$, so it follows from Theorem $21(1)$ that $j_{1,12}(\sqrt{-2} / 2)$ generates $K_{\mathfrak{f}}$ over $K$ with $\mathfrak{f}=3[2, \sqrt{-2}]^{3}$.

Take $K=\mathbb{Q}(\sqrt{-7})$ and $\mathfrak{a}=[2,(-1+\sqrt{-7}) / 2]$. Then $d_{K}=-7 \equiv 1$ $(\bmod 8)$, so it follows from Theorem $21(1)$ that $j_{1,12}((-1+\sqrt{-7}) / 4)$ generates $K_{\mathrm{f}}$ with

$$
\mathfrak{f}=3\left[2, \frac{-1+\sqrt{-7}}{2}\right]\left[2, \frac{1+\sqrt{-7}}{2}\right]^{2} .
$$

(2) Take $K=\mathbb{Q}(\sqrt{-21})$ and $\mathfrak{a}=[21, \sqrt{-21}]$. Then $d_{K}=-4 \cdot 21 \equiv 0$ $(\bmod 3)$, so it follows from Theorem $21(2)$ that $j_{1,12}(\sqrt{-21} / 21)$ generates $K_{\mathfrak{f}}$ over $K$ with $\mathfrak{f}=4[3, \sqrt{-21}]$.

(3) Take $K=\mathbb{Q}(\sqrt{-6})$ and $\mathfrak{a}=[6, \sqrt{-6}]$. Then $d_{K}=-4 \cdot 6 \equiv 0$ $(\bmod 6)$, so it follows from Theorem $21(4)$ that $j_{1,12}(\sqrt{-6} / 6)$ generates $K_{\mathfrak{f}}$ over $K$ with $\mathfrak{f}=[2, \sqrt{-6}]^{3}[3, \sqrt{-6}]$.

Take $K=\mathbb{Q}(\sqrt{-15})$ and $\mathfrak{a}=[6,(-3+\sqrt{-15}) / 2]$. Then $\alpha=(-3+$ $\sqrt{-15}) / 12$ satisfies the equation $6 X^{2}+3 X+1=0$, so it follows from Theorem $21(4)$ that $j_{1,12}((-3+\sqrt{-15}) / 12)$ generates $K_{\mathfrak{f}}$ over $K$ with

$$
\mathfrak{f}=\left[2, \frac{1+\sqrt{-15}}{2}\right]\left[2, \frac{-1+\sqrt{-15}}{2}\right]^{2}\left[3, \frac{-3+\sqrt{-15}}{2}\right] .
$$

In Theorem 21, we constructed ray class fields $K_{\mathfrak{f}}$ with conductor $\mathfrak{f}$ which strictly divide 12 under the assumption $K \neq \mathbb{Q}(\sqrt{-1}), \mathbb{Q}(\sqrt{-3})$. As we saw in the course of proof, however, a crucial point making its proof formidable was the nontriviality of $\Gamma_{\alpha}$ when $K=\mathbb{Q}(\sqrt{-1}), \mathbb{Q}(\sqrt{-3})$. We now give other descriptions for spanning $K_{\mathfrak{f}}$ in these cases by a thorough analysis of $\Gamma_{\alpha}$.

TheOREM 23. Notations being as in Theorem 21 except for the discriminant, we have the following assertions:

(1) If $(a, 12)=2$, then $j_{1,12}(\alpha)$ generates $\mathbb{Q}(\sqrt{-1})_{\mathfrak{f}}$ over $\mathbb{Q}(\sqrt{-1})$ with conductor $\mathfrak{f}=3[2, a \alpha]^{3}$. In this case, 2 ramifies in $\mathbb{Q}(\sqrt{-1})$ as $[2, a \alpha]^{2}$, and so we have $12 \mathcal{O}_{K}=3[2, a \alpha]^{4}$.

(2) If $(a, 12)=3$, then $j_{1,12}(\alpha)$ generates $\mathbb{Q}(\sqrt{-3})_{\mathfrak{f}}$ over $\mathbb{Q}(\sqrt{-3})$ with conductor $\mathfrak{f}=4[3, a \alpha]$. Furthermore, 3 ramifies in $\mathbb{Q}(\sqrt{-3})$ as $[3, a \alpha]^{2}$, and hence $12 \mathcal{O}_{K}=4[3, a \alpha]^{2}$.

REMARK 24. (1) In the case $(a, 12)=2$ and $K=\mathbb{Q}(\sqrt{-3})$, we see that there is no $\alpha$ satisfying the hypothesis. For, otherwise, $b^{2}-4 a c=-3$ implies that $b^{2} \equiv 5(\bmod 8)$, which is absurd. 
(2) In the case $(a, 12)=3$ and $K=\mathbb{Q}(\sqrt{-1})$, no such $\alpha$ exists. Indeed, otherwise, $b^{2}-4 a c=-4$ implies that $b^{2} \equiv 8(\bmod 12)$, which is impossible, too.

(3) In a similar way, in the cases $(a, 12)=4,6$ and 12 , we see that there exists no such $\alpha$ for both fields $\mathbb{Q}(\sqrt{-1})$ and $\mathbb{Q}(\sqrt{-3})$.

Pro of (of Theorem 23). (1) The arguments from the beginning to the nontriviality of $\Gamma_{\alpha}$ are exactly the same as those in Theorem 21. Suppose that $\alpha$ is equivalent to $i$ under $\mathrm{SL}_{2}(\mathbb{Z})$, in which case $d_{K}=-4$. Put $\mathfrak{f}=$ $6[2, a \alpha]$. Then we have, for $(\beta) \in I_{K}(12)$,

$$
\begin{aligned}
(\beta) \in P_{K, 1}(\mathfrak{f}) \Leftrightarrow & \pm \beta \equiv 1(\bmod \mathfrak{f}) \text { or } \pm \beta i \equiv 1(\bmod \mathfrak{f}) \\
\Leftrightarrow & \pm \beta \in 1+6[2, a \alpha] \text { or } \\
& 6 \mid\left(\frac{-b}{2} n+m\right) \text { and } \frac{b}{2}\left(m-\frac{b}{2} n\right)-n \equiv \pm 1(\bmod 12) .
\end{aligned}
$$

Here, the second statement is due to the fact that $a \alpha=-b / 2+i$ and $b^{2}-4 a c=-4$. On the other hand,

$$
\begin{aligned}
(\beta) \in \operatorname{Ker}\left(\Phi_{K\left(j_{1,12}(\alpha)\right) / K}\right) \Leftrightarrow & \mathcal{A}_{\beta} \in \pm \Gamma_{1}(12) \cdot \Gamma_{\alpha} \\
\Leftrightarrow & \mathcal{A}_{\beta} \in \pm \Gamma_{1}(12) \text { or } \\
& \mathcal{A}_{\beta} \cdot\left(\gamma^{-1}\left(\begin{array}{cc}
0 & -1 \\
1 & 0
\end{array}\right) \gamma\right) \in \pm \Gamma_{1}(12),
\end{aligned}
$$

where $\alpha=\gamma^{-1} i$ for some $\gamma=\left(\begin{array}{ll}p & q \\ r & s\end{array}\right) \in \mathrm{SL}_{2}(\mathbb{Z})$. Since $\alpha$ is the root of the polynomial $[1,0,1] \circ\left(\begin{array}{cc}p & q \\ r & s\end{array}\right)\left(\begin{array}{l}z \\ 1\end{array}\right)=\left(p^{2}+r^{2}\right) z^{2}+2(p q+r s) z+\left(q^{2}+s^{2}\right)$, we get $a=p^{2}+r^{2}, b=2(p q+r s)$ and $c=q^{2}+s^{2}$. Thus we get

$$
\gamma^{-1}\left(\begin{array}{cc}
0 & -1 \\
1 & 0
\end{array}\right) \gamma=\left(\begin{array}{cc}
-(p q+r s) & -\left(q^{2}+s^{2}\right) \\
p^{2}+r^{2} & p q+r s
\end{array}\right)=\left(\begin{array}{cc}
-b / 2 & -c \\
a & b / 2
\end{array}\right) .
$$

Therefore,

$$
\begin{aligned}
\mathcal{A}_{\beta} \cdot\left(\gamma^{-1}\left(\begin{array}{cc}
0 & -1 \\
1 & 0
\end{array}\right) \gamma\right) & =\left(\begin{array}{cc}
-b n+m & -c n \\
a n N(\beta)^{-1} & m N(\beta)^{-1}
\end{array}\right)\left(\begin{array}{cc}
-b / 2 & -c \\
a & b / 2
\end{array}\right) \\
& =\left(\begin{array}{cc}
b^{2} n / 2-b m / 2-a c n & * \\
(-a b n / 2+a m) N(\beta)^{-1} & *
\end{array}\right),
\end{aligned}
$$

where

$$
\frac{b^{2} n}{2}-\frac{b m}{2}-a c n=-\frac{b}{2}\left(m-\frac{b}{2} n\right)-n
$$

Then we have

$$
\begin{aligned}
\mathcal{A}_{\beta} \in & \pm \Gamma_{1}(12) \text { or } \mathcal{A}_{\beta} \cdot\left(\gamma^{-1}\left(\begin{array}{cc}
0 & -1 \\
1 & 0
\end{array}\right) \gamma\right) \in \pm \Gamma_{1}(12) \\
\Leftrightarrow & 12 \mid \text { an }, m \in \pm 1+b n+12 \mathbb{Z}, \text { or } \\
& 12 \mid a\left(m-\frac{b}{2} n\right) \text { and }-\frac{b}{2}\left(m-\frac{b}{2} n\right)-n \equiv \pm 1(\bmod 12)
\end{aligned}
$$




$$
\begin{aligned}
\Leftrightarrow & 6 \mid n, \pm \beta \in 1+n(a \alpha+b)+12 \mathbb{Z}, \text { or } \\
& 6 \mid\left(m-\frac{b}{2} n\right) \text { and }-\frac{b}{2}\left(m-\frac{b}{2} n\right)-n \equiv \pm 1(\bmod 12) \\
\Leftrightarrow & \pm \beta \in 1+6[2, a \alpha+b]=1+6[2, a \alpha], \text { or } \\
& 6 \mid\left(m-\frac{b}{2} n\right) \text { and } \frac{b}{2}\left(m-\frac{b}{2} n\right)-n \equiv \pm 1(\bmod 12) .
\end{aligned}
$$

Consequently, we see that $(\beta) \in \operatorname{Ker}\left(\Phi_{K\left(j_{1,12}(\alpha)\right) / K}\right) \Leftrightarrow(\beta) \in P_{K, 1}(\mathfrak{f}) \cap$ $I_{K}(12)$, and the result follows.

(2) Assume that $\alpha$ is equivalent to $\varrho$ under $\mathrm{SL}_{2}(\mathbb{Z})$, in which case $d_{K}=$ -3 . Since $\Gamma_{\varrho}=\left\{ \pm I_{2}, \pm\left(\begin{array}{cc}0 & -1 \\ 1 & 1\end{array}\right), \pm\left(\begin{array}{cc}1 & 1 \\ -1 & 0\end{array}\right)\right\}$, we see that

$$
\Gamma_{\alpha}=\left\{ \pm I_{2}, \pm \gamma^{-1}\left(\begin{array}{cc}
0 & -1 \\
1 & 1
\end{array}\right) \gamma, \pm \gamma^{-1}\left(\begin{array}{cc}
1 & 1 \\
-1 & 0
\end{array}\right) \gamma\right\}
$$

for some $\gamma \in \mathrm{SL}_{2}(\mathbb{Z})$. Put $\mathfrak{f}=4[3, a \alpha]$. Then we have, for $(\beta) \in I_{K}(12)$,

$(\beta) \in P_{K, 1}(\mathfrak{f}) \Leftrightarrow \pm \beta \equiv 1(\bmod \mathfrak{f})$ or $\pm \beta \varrho \equiv 1(\bmod \mathfrak{f})$

$$
\text { or } \pm \beta \varrho^{2} \equiv 1(\bmod \mathfrak{f})
$$

$\Leftrightarrow \pm \beta \in 1+4[3, a \alpha]$, or

$$
\begin{aligned}
& 4 \mid\left(\frac{b+1}{2} n-m\right) \text { and } \frac{b-1}{2} m-\frac{b^{2}+3}{4} n \equiv \pm 1(\bmod 12) \text {, or } \\
& 4 \mid\left(\frac{b-1}{2} n-m\right) \text { and }-\frac{b+1}{2} m+\frac{b^{2}+3}{4} n \equiv \pm 1(\bmod 12) .
\end{aligned}
$$

Here, the second argument is due to the fact that $\varrho=a \alpha+(b-1) / 2$, $\varrho^{2}=-a \alpha-(b+1) / 2$ and $b^{2}-4 a c=-3$. On the other hand,

$$
\begin{aligned}
(\beta) \in \operatorname{Ker}\left(\Phi_{K\left(j_{1,12}(\alpha)\right) / K}\right) \Leftrightarrow & \mathcal{A}_{\beta} \in \pm \Gamma_{1}(12) \cdot \Gamma_{\alpha} \\
\Leftrightarrow & \mathcal{A}_{\beta} \in \pm \Gamma_{1}(12) \text { or } \mathcal{A}_{\beta} \cdot\left(\gamma^{-1}\left(\begin{array}{cc}
1 & 1 \\
-1 & 0
\end{array}\right) \gamma\right) \in \pm \Gamma_{1}(12) \\
& \text { or } \mathcal{A}_{\beta} \cdot\left(\gamma^{-1}\left(\begin{array}{cc}
0 & -1 \\
1 & 1
\end{array}\right) \gamma\right) \in \pm \Gamma_{1}(12)
\end{aligned}
$$

where $\alpha=\gamma^{-1} \varrho$ for some $\gamma=\left(\begin{array}{ll}p & q \\ r & s\end{array}\right) \in \mathrm{SL}_{2}(\mathbb{Z})$. Since $\alpha$ is the root of the polynomial $[1,1,1] \circ\left(\begin{array}{cc}p & q \\ r & s\end{array}\right)\left(\begin{array}{l}z \\ 1\end{array}\right)=\left(p^{2}+p r+r^{2}\right) z^{2}+(2 p q+p s+r q+$ $2 r s) z+\left(q^{2}+q s+s^{2}\right)$, we get $a=p^{2}+p r+r^{2}, b=2 p q+p s+r q+2 r s$ $(=2(p q+p s+r s)-1=2(p q+r q+r s)+1)$ and $c=q^{2}+q s+s^{2}$. Thus

$$
\begin{aligned}
\gamma^{-1}\left(\begin{array}{cc}
1 & 1 \\
-1 & 0
\end{array}\right) \gamma & =\left(\begin{array}{cc}
p s+p q+r s & q^{2}+s q+s^{2} \\
-\left(p^{2}+r p+r^{2}\right) & -(q r+p q+r s)
\end{array}\right) \\
& =\left(\begin{array}{cc}
(b+1) / 2 & c \\
-a & -(b-1) / 2
\end{array}\right),
\end{aligned}
$$


and

$$
\begin{aligned}
\mathcal{A}_{\beta} \cdot\left(\gamma^{-1}\left(\begin{array}{cc}
1 & 1 \\
-1 & 0
\end{array}\right) \gamma\right) \\
\quad=\left(\begin{array}{cc}
-b n+m & -c n \\
a n N(\beta)^{-1} & m N(\beta)^{-1}
\end{array}\right)\left(\begin{array}{cc}
(b+1) / 2 & c \\
-a & -(b-1) / 2
\end{array}\right) \\
\quad=\left(\begin{array}{cc}
\frac{b+1}{2}(-b n+m)+a c n & * \\
\left(\frac{b+1}{2} n-m\right) a N(\beta)^{-1} & *
\end{array}\right),
\end{aligned}
$$

where

$$
\frac{b+1}{2}(-b n+m)+a c n=-b\left(\frac{b+1}{2} n-m\right)-\frac{b-1}{2} m+\frac{b^{2}+3}{4} n .
$$

In the same manner, we have

$$
\begin{aligned}
\gamma^{-1}\left(\begin{array}{cc}
0 & -1 \\
1 & 1
\end{array}\right) \gamma & =\left(\begin{array}{cc}
-(p q+r s+r q) & -\left(q^{2}+s q+s^{2}\right) \\
p^{2}+r p+r^{2} & p q+p s+r s
\end{array}\right) \\
& =\left(\begin{array}{cc}
-(b-1) / 2 & -c \\
a & (b+1) / 2
\end{array}\right)
\end{aligned}
$$

and

where

$$
\mathcal{A}_{\beta} \cdot\left(\gamma^{-1}\left(\begin{array}{cc}
0 & -1 \\
1 & 1
\end{array}\right) \gamma\right)=\left(\begin{array}{ll}
-\frac{b-1}{2}(-b n+m)-a c n & * \\
\left(-\frac{b-1}{2} n+m\right) a N(\beta)^{-1} & *
\end{array}\right),
$$

$$
-\frac{b-1}{2}(-b n+m)-a c n=b\left(\frac{b-1}{2} n-m\right)+\frac{b+1}{2} m-\frac{b^{2}+3}{4} n .
$$

So we get

$$
\begin{aligned}
\mathcal{A}_{\beta} \in & \pm \Gamma_{1}(12) \text { or } \mathcal{A}_{\beta} \cdot\left(\gamma^{-1}\left(\begin{array}{cc}
1 & 1 \\
-1 & 0
\end{array}\right) \gamma\right) \in \pm \Gamma_{1}(12) \\
& \text { or } \mathcal{A}_{\beta} \cdot\left(\gamma^{-1}\left(\begin{array}{cc}
0 & -1 \\
1 & 1
\end{array}\right) \gamma\right) \in \pm \Gamma_{1}(12) \\
\Leftrightarrow & 12 \mid a n, m \in \pm 1+b n+12 \mathbb{Z}, \text { or } 12 \mid a\left(\frac{b+1}{2} n-m\right) \text { and } \\
& -b\left(\frac{b+1}{2} n-m\right)-\frac{b-1}{2} m+\frac{b^{2}+3}{4} n \equiv \pm 1(\bmod 12), \text { or } \\
& 12 \mid a\left(-\frac{b-1}{2} n+m\right) \text { and } b\left(\frac{b-1}{2} n-m\right)+\frac{b+1}{2} m-\frac{b^{2}+3}{4} n \\
& \equiv \pm 1(\bmod 12) \\
\Leftrightarrow & 4 \mid n, m \in \pm 1+b n+12 \mathbb{Z}, \text { or } 4 \mid\left(\frac{b+1}{2} n-m\right) \text { and }
\end{aligned}
$$




$$
\begin{aligned}
& -b\left(\frac{b+1}{2} n-m\right)-\frac{b-1}{2} m+\frac{b^{2}+3}{4} n \equiv \pm 1(\bmod 12), \text { or } \\
& 4 \mid\left(-\frac{b-1}{2} n+m\right) \text { and } b\left(\frac{b-1}{2} n-m\right)+\frac{b+1}{2} m-\frac{b^{2}+3}{4} n \\
& \equiv \pm 1(\bmod 12) \\
\Leftrightarrow & \pm \beta \in 1+4[3, a \alpha+b]=1+4[3, a \alpha], \text { or } \\
& 4 \mid\left(\frac{b+1}{2} n-m\right) \text { and }-\frac{b-1}{2} m+\frac{b^{2}+3}{4} n \equiv \pm 1(\bmod 12), \text { or } \\
& 4 \mid\left(-\frac{b-1}{2} n+m\right) \text { and } \frac{b+1}{2} m-\frac{b^{2}+3}{4} n \equiv \pm 1(\bmod 12) .
\end{aligned}
$$

Therefore, we see that

$$
(\beta) \in \operatorname{Ker}\left(\Phi_{K\left(j_{1,12}(\alpha)\right) / K}\right) \Leftrightarrow(\beta) \in P_{K, 1}(\mathfrak{f}) \cap I_{K}(12),
$$

and the theorem follows.

Examples. (1) Take $K=\mathbb{Q}(\sqrt{-1})$ and $\mathfrak{a}=[1,(1+\sqrt{-1}) / 2]$. Then $\alpha=(1+\sqrt{-1}) / 2$ satisfies $2 X^{2}-2 X+1=0$. It follows from Theorem 23(1) that $j_{1,12}((1+\sqrt{-1}) / 2)$ generates $\mathbb{Q}(\sqrt{-1})_{\mathfrak{f}}$ over $\mathbb{Q}(\sqrt{-1})$ with conductor $\mathfrak{f}=3[2,1+\sqrt{-1}]^{3}$.

(2) Take $K=\mathbb{Q}(\sqrt{-3})$ and $\mathfrak{a}=[3,(-3+\sqrt{-3}) / 2]$. Then $\alpha=(-3+$ $\sqrt{-3}) / 6$ satisfies $3 X^{2}+3 X+1=0$. We are certain by Theorem $23(2)$ that $j_{1,12}((-3+\sqrt{-3}) / 6)$ generates $\mathbb{Q}(\sqrt{-3})_{\mathfrak{f}}$ over $\mathbb{Q}(\sqrt{-3})$ with conductor $\mathfrak{f}=4[3,(-3+\sqrt{-3}) / 2]$.

Table 1. Conductor $\mathfrak{f}$ of $K\left(j_{1,12}(\alpha)\right)$ $(\times$ means that there is no $\alpha$ satisfying the condition)

\begin{tabular}{|c|c|c|c|}
\hline & $(a, 12)=1$ & $(a, 12)=2$ & $(a, 12)=4$ \\
\hline$d_{K} \equiv 0(\bmod 4)$ & $(12)$ & $3[2, a \alpha]^{3}$ & $\times$ \\
\hline$d_{K} \equiv 1(\bmod 8)$ & $(12)$ & $3[2, a \alpha][2, a \alpha+1]^{2}$ & $3[2, a \alpha+1]^{2}$ \\
\hline$d_{K} \equiv 5(\bmod 8)$ & $(12)$ & $\times$ & $\times$ \\
\hline
\end{tabular}

\begin{tabular}{|c|c|c|c|}
\hline & $(a, 12)=3$ & $\begin{array}{c}(a, 12)=12, \\
d_{K} \equiv 1(\bmod 8)\end{array}$ & $\begin{array}{c}(a, 12)=12, \\
d_{K} \neq \equiv 1(\bmod 8)\end{array}$ \\
\hline$b \equiv 0(\bmod 3)$ & $4[3, a \alpha]$ & {$[2, a \alpha+1]^{2}[3, a \alpha]$} & $\times$ \\
\hline$b \equiv 1(\bmod 3)$ & $4[3, a \alpha+1]$ & {$[2, a \alpha+1]^{2}[3, a \alpha+1]$} & $\times$ \\
\hline$b \equiv 2(\bmod 3)$ & $4[3, a \alpha+2]$ & {$[2, a \alpha+1]^{2}[3, a \alpha+2]$} & $\times$ \\
\hline
\end{tabular}


Table 1 (cont.)

\begin{tabular}{|c|c|c|}
\hline & $\begin{array}{c}(a, 12)=6, \\
d_{K} \neq \equiv(\bmod 8)\end{array}$ & $\begin{array}{c}(a, 12)=6, \\
d_{K} \equiv 5(\bmod 8)\end{array}$ \\
\hline$b \equiv 0(\bmod 6)$ & {$[2, a \alpha]^{3}[3, a \alpha]$} & $\times$ \\
\hline$b \equiv 1(\bmod 6)$ & {$[2, a \alpha][2, a \alpha+1]^{2}[3, a \alpha+1]$} & $\times$ \\
\hline$b \equiv 2(\bmod 6)$ & {$[2, a \alpha]^{3}[3, a \alpha+2]$} & $\times$ \\
\hline$b \equiv 3(\bmod 6)$ & {$[2, a \alpha][2, a \alpha+1]^{2}[3, a \alpha]$} & $\times$ \\
\hline$b \equiv 4(\bmod 6)$ & {$[2, a \alpha]^{3}[3, a \alpha+1]$} & $\times$ \\
\hline$b \equiv 5(\bmod 6)$ & {$[2, a \alpha][2, a \alpha+1]^{2}[3, a \alpha+2]$} & $\times$ \\
\hline
\end{tabular}

6. Explicit calculation of minimal polynomials. In this section, we will find an explicit formula for the conjugates of $j_{1,12}(\alpha)$ permitting the numerical calculation of its minimal polynomial. Since $t(\alpha):=N\left(j_{1,12}(\alpha)\right)$ is an algebraic integer ([11], Corollary 7$)$, it is more convenient to work with $t$ than with $j_{1,12}$ in realizing its minimal polynomial. Let $\mathcal{Q}_{d_{K}}(N)$ be the set of primitive quadratic forms $\left[a^{\prime}, b^{\prime}, c^{\prime}\right]$ having discriminant $d_{K}$ with conditions $a^{\prime}>0$ and $\left(a^{\prime}, N\right)=1$. For $\gamma \in \Gamma_{0}(N)$ and $\mathcal{Q} \in \mathcal{Q}_{d_{K}}(N)$, $\mathcal{Q} \circ \gamma$ again belongs to $\mathcal{Q}_{d_{K}}(N)$. Hence the quotients $\mathcal{Q}_{d_{K}}(N) / \Gamma_{0}(N)$ and $\mathcal{Q}_{d_{K}}(N) / \Gamma_{1}(N)$ are well defined.

TheOREM 25. With $K, \mathfrak{a}$ and $\alpha$ as before, let $a z^{2}+b z+c=0$ be the equation of $\alpha$ such that $a>0$ and $(a, b, c)=1$. Suppose that $(a, 12)=1$. Then:

(1) $\left|\mathcal{Q}_{d_{K}}(12) / \Gamma_{1}(12)\right|=2 h(\mathcal{O})$, where $\mathcal{O}=\mathbb{Z}+12 \mathcal{O}_{K}$ and $h(\mathcal{O})$ denotes the class number of $\mathcal{O}$. Set

(2) Let $\left\{\mathcal{Q}_{i}\right\}_{i=1}^{2 h(\mathcal{O})}$ be a complete set of representatives for $\mathcal{Q}_{d_{K}}(12) / \Gamma_{1}(12)$.

$$
f(X)=\prod_{i=1}^{2 h(\mathcal{O})}\left(X-t\left(\tau_{\mathcal{Q}_{i}}\right)\right) .
$$

Then $f(X)$ is the minimal polynomial of $t(\alpha)$ over $K$. Here, $\tau_{\mathcal{Q}_{i}}$ denotes the root of the equation $\mathcal{Q}_{i}(z, 1)=0$ in $\mathfrak{H}$. Moreover, $f(X) \in \mathbb{Z}[X]$.

Proof. First, we recall from [1], Proposition 4.1, that there is a one-toone correspondence between $\mathcal{Q}_{d_{K}}(12) / \Gamma_{0}(12)$ and $I_{K}(12) / P_{K, \mathbb{Z}}(12)$, which maps $[a, b, c] \in \mathcal{Q}_{d_{K}}(12) / \Gamma_{0}(12)$ to $\left[a,\left(-b+\sqrt{d_{K}}\right) / 2\right] \in I_{K}(12) / P_{K, \mathbb{Z}}(12)$. Hence the cardinality of $\mathcal{Q}_{d_{K}}(12) / \Gamma_{0}(12)$ is equal to $h(\mathcal{O})$ because

$$
I_{K}(12) / P_{K, \mathbb{Z}}(12) \cong \operatorname{Gal}(L / K),
$$

where $L$ is the ring class field of $\mathcal{O}=\mathbb{Z}+12 \mathcal{O}_{K}$ over $K$.

Now let $\pi: \mathcal{Q}_{d_{K}}(12) / \Gamma_{1}(12) \rightarrow \mathcal{Q}_{d_{K}}(12) / \Gamma_{0}(12)$ be the natural projection. Choose an element $\gamma$ in $\Gamma_{0}(12) \backslash \pm \Gamma_{1}(12)$, and consider the decomposi- 
tion $\bar{\Gamma}_{0}(12)=\bar{\Gamma}_{1}(12) \cup \gamma \bar{\Gamma}_{1}(12)$ as transformation groups. It can be easily shown that $\pi^{-1}(\mathcal{Q})=\{\mathcal{Q}, \mathcal{Q} \circ \gamma\}$ for each $\mathcal{Q} \in \mathcal{Q}_{d_{K}}(12) / \Gamma_{0}(12)$. We claim that $\mathcal{Q}$ cannot be equivalent to $\mathcal{Q} \circ \gamma$ under $\Gamma_{1}(12)$. Indeed, if $\mathcal{Q} \sim \mathcal{Q} \circ \gamma$ under $\Gamma_{1}(12)$, then $\mathcal{Q}=\mathcal{Q} \circ \gamma^{\prime}$ for some $\gamma^{\prime} \in \Gamma_{0}(12) \backslash \pm \Gamma_{1}(12)$. Let $\tau_{\mathcal{Q}}(\in \mathfrak{H})$ be the root of $\mathcal{Q}(z, 1)=0$. Then $\gamma^{\prime-1} \tau_{\mathcal{Q}}$ is the root of $\mathcal{Q} \circ \gamma^{\prime}$ in $\mathfrak{H}$ and it must be equal to $\tau_{\mathcal{Q}}$. On the other hand, we see that $\Gamma_{0}(12)$ has no elliptic element ([19], Proposition 1.43). Thus $\gamma^{\prime}$ turns out to be trivial, which is a contradiction. This proves (1).

We note that the order $\mathcal{O}_{\mathfrak{a}}$ of an $\mathcal{O}_{K}$-ideal $\mathfrak{a}$ is $\mathcal{O}_{K}$ itself. Since $\mathcal{O}_{\mathfrak{a}}=$ $\mathcal{O}_{K}=[1, a \alpha], b^{2}-4 a c=d_{K}<0,(a, 12)=1$ and $(a, b, c)=1,[a, b, c]$ belongs to $\mathcal{Q}_{d_{K}}(12)$. Hence $t(\alpha)=t\left(\tau_{\mathcal{Q}_{i}}\right)$ for some $i$. So $f(X)$ certainly has $t(\alpha)$ as a root. Now we claim that the conjugate of $t(\alpha)$ over $K$ must be of the form $t\left(\tau^{\prime}\right)$, where $\tau^{\prime}$ is a root of a quadratic form $\left[a^{\prime}, b^{\prime}, c^{\prime}\right] \in \mathcal{Q}_{d_{K}}(12)$. Indeed, let $\sigma$ be an embedding of $K_{(12)}$ over $K$. Then there exists an ideal $\mathfrak{a} \in I_{K}(12)$ such that $\sigma=\left[\mathfrak{a}, K_{(12)} / K\right]$. Since $t$ has rational coefficients, we get

$$
t(\alpha)^{\sigma}=t(\alpha)^{\left[\mathfrak{a}, K_{(12)} / K\right]}=t(\mathcal{A} \cdot \alpha)
$$

for some $\mathcal{A} \in G_{\mathbb{Q}+}([1],(3.7 .3))$. Since $T_{12 I}=N\left(j_{0,12}\right)$ is a rational function of $t$, it follows that $T_{12 I}^{\sigma}=T_{12 I}\left(\tau^{\prime}\right)$, where $\tau^{\prime}=\mathcal{A} \cdot \alpha$. Define $\operatorname{disc}\left(\tau^{\prime}\right)=$ $\operatorname{disc} \mathcal{O}_{\left[1, \tau^{\prime}\right]}=b^{\prime 2}-4 a^{\prime} c^{\prime}$, where $a^{\prime} \tau^{\prime 2}+b^{\prime} \tau^{\prime}+c^{\prime}=0, a^{\prime}>0$ and $\left(a^{\prime}, b^{\prime}, c^{\prime}\right)=1$. Assume that $\mathcal{A}=\left(\begin{array}{ll}p & q \\ r & s\end{array}\right) \in M_{2}(\mathbb{Z})$ with $(p, q, r, s)=1$. Put $\operatorname{disc}\left(\tau^{\prime}\right)=m^{2} d_{K}$. Now, by Theorem 3.7.5(1) of [1], $K\left(T_{12 I}\left(\tau^{\prime}\right)\right)$ is the ring class field of an order $\mathcal{O}^{\prime}=\mathbb{Z}+f \mathcal{O}_{K}$, where $f=m \cdot 12 /\left(a^{\prime}, 12\right)$. On the other hand, $K\left(T_{12 I}(\alpha)\right)$ is the ring class field of $\mathcal{O}=\mathbb{Z}+12 \mathcal{O}_{K}$. Since $T_{12 I}\left(\tau^{\prime}\right)$ is a conjugate of $T_{12 I}(\alpha)$, the two fields $K\left(T_{12 I}\left(\tau^{\prime}\right)\right)$ and $K\left(T_{12 I}(\alpha)\right)$ coincide, so that $m=\left(a^{\prime}, 12\right)$. Let $\mathcal{A}^{\iota}=\left(\begin{array}{cc}s & -q \\ -r & p\end{array}\right)$ be the main involution of $\mathcal{A}$ and $\mathcal{Q} \circ \mathcal{A}^{\iota}(z, 1)=a^{\prime \prime} z^{2}+b^{\prime \prime} z+c^{\prime \prime}$, where $\mathcal{Q}=[a, b, c]$. Since $\tau^{\prime}=\mathcal{A} \cdot \alpha$ is a root of the polynomial $\mathcal{Q} \circ \mathcal{A}^{\iota}(z, 1)$ and $a^{\prime \prime}$ is positive, it follows that $\mathcal{Q} \circ \mathcal{A}^{\iota}(z, 1) /\left(a^{\prime \prime}, b^{\prime \prime}, c^{\prime \prime}\right)=a^{\prime} z^{2}+b^{\prime} z+c^{\prime}$. By taking discriminants on both sides, we get $\operatorname{det}(\mathcal{A})^{2} \cdot d_{K}=\left(a^{\prime \prime}, b^{\prime \prime}, c^{\prime \prime}\right)^{2} \cdot m^{2} \cdot d_{K}$, so that $m \operatorname{divides} \operatorname{det}(\mathcal{A})$. But $(N(\mathfrak{a}), 12)=1$ implies that $\left(\operatorname{det}\left(\xi_{\alpha}\left(\mathfrak{s}^{-1}\right)\right), 12\right)=1$, where $\mathfrak{s}$ is an idele corresponding to $\mathfrak{a}$. Thus $(\operatorname{det}(\mathcal{A}), 12)=1$ and so $(m, 12)=1$. Since $m=$ $\left(a^{\prime}, 12\right)$, both $m$ and $\left(a^{\prime}, 12\right)$ must be 1 . This shows that $\left[a^{\prime}, b^{\prime}, c^{\prime}\right] \in \mathcal{Q}_{d_{K}}(12)$ and $t\left(\tau^{\prime}\right)=t\left(\tau_{\mathcal{Q}_{j}}\right)$ for some $j$. Since $\left|\mathcal{Q}_{d_{K}}(12) / \Gamma_{1}(12)\right|=2 h(\mathcal{O})$ and there are exactly $2 h(\mathcal{O})$ conjugates of $t(\alpha)$ (Theorem $20(2)$ ), the first part of the assertion (2) is proved.

For the second part of $(2)$, let $t(z)=q^{-1}+\sum_{n>1} H_{n} q^{n}\left(H_{n} \in \mathbb{Z}\right)$ be the Fourier expansion of $t$. Write $\tau_{\mathcal{Q}}=x+i y \in \mathfrak{H}$ and consider

$$
\begin{aligned}
\overline{t\left(\tau_{\mathcal{Q}}\right)} & =\overline{e^{-2 \pi i(x+i y)}}+\sum_{n \geq 1} H_{n} \overline{e^{2 \pi i n(x+i y)}}=e^{-2 \pi i(-x+i y)}+\sum_{n \geq 1} H_{n} e^{2 \pi i n(-x+i y)} \\
& =t(-x+i y)=t\left(\tau_{\overline{\mathcal{Q}}}\right)
\end{aligned}
$$


where $\overline{\mathcal{Q}}$ is defined to be $[a,-b, c]$ when $\mathcal{Q}=[a, b, c]$. Hence the complex conjugate fixes the roots of $f(X)$ and so $f(X) \in \mathbb{R}[X]$. But, since $t(\alpha)$ is an algebraic integer and $K$ is an imaginary quadratic field, $f(X)$ lies in $\left(\mathbb{R} \cap \mathcal{O}_{K}\right)[X]=\mathbb{Z}[X]$.

Example. Take $K=\mathbb{Q}(\sqrt{-1})$ and $\mathfrak{a}=[1, \sqrt{-1}]=\mathcal{O}_{K}$. Then the degree of $K\left(j_{1,12}(\sqrt{-1})\right)$ over $K$ is $2 h\left(\mathbb{Z}+12 \mathcal{O}_{K}\right)=16$. Observe that

$$
\begin{aligned}
& \mathcal{Q}_{d_{K}}(12) / \Gamma_{0}(12)=\{[1,0,1],[5,4,1],[5,6,2],[17,8,1] \\
& {[17,-8,1],[13,10,2],[37,12,1],[25,14,2]\} . }
\end{aligned}
$$

For any $\gamma \in \Gamma_{0}(12) \backslash \pm \Gamma_{1}(12)$, we have

$$
\mathcal{Q}_{d_{K}}(12) / \Gamma_{1}(12)=\left\{\mathcal{Q}, \mathcal{Q} \circ \gamma \mid \mathcal{Q} \in \mathcal{Q}_{d_{K}}(12) / \Gamma_{0}(12)\right\} .
$$

Now Theorem 25(2) permits an explicit calculation of the minimal polynomial of $t(\sqrt{-1})=N\left(j_{1,12}(\sqrt{-1})\right)$. In fact, by approximating $t\left(\tau_{\mathcal{Q}_{i}}\right)$ with the aid of computer, we can determine the coefficients of $f(X)=\prod_{i}\left(X-t\left(\tau_{\mathcal{Q}_{i}}\right)\right)$ because we already know that $f(X)$ is in $\mathbb{Z}[X]$. Taking the representatives of $\mathcal{Q}_{d_{K}}(12) / \Gamma_{0}(12)$ as above and $\gamma=\left(\begin{array}{cc}7 & 4 \\ 12 & 7\end{array}\right) \in \Gamma_{0}(12) \backslash \pm \Gamma_{1}(12)$, we see that the minimal polynomial of $t(\sqrt{-1})$ is

$$
\begin{aligned}
X^{16}- & 520 X^{15}-8184 X^{14}-59840 X^{13}-266800 X^{12}-813984 X^{11} \\
& -1810976 X^{10}-3051904 X^{9}-3978144 X^{8}-4039552 X^{7}-317504 X^{6} \\
& -1886208 X^{5}-803584 X^{4}-218624 X^{3}-26112 X^{2}+2048 X+256 .
\end{aligned}
$$

Theorem 26. Let $K, \mathfrak{a}$ and $\alpha$ be as in Theorem 25. Assume that $(a, 12)=$ 2 and $d_{K} \equiv 0(\bmod 4) . \quad$ Let $\mathcal{Q}_{d_{K}}^{(2)}=\left\{\left[a^{\prime}, b^{\prime}, c^{\prime}\right] \in \mathcal{Q}_{d_{K}} \mid\left(a^{\prime}, 12\right)=2\right\}$, where $\mathcal{Q}_{d_{K}}$ is the set of positive definite primitive quadratic forms having discriminant $d_{K}$. Then the quotient $\mathcal{Q}_{d_{K}}^{(2)} / \Gamma_{1}(12)$ is well defined and its cardinality is equal to the class number $h(\mathcal{O})$ of the order $\mathcal{O}=\mathbb{Z}+12 \mathcal{O}_{K}$. Let $\left\{\mathcal{Q}_{i}\right\}_{i=1}^{h(\mathcal{O})}$ be a complete set of representatives for $\mathcal{Q}_{d_{K}}^{(2)} / \Gamma_{1}(12)$ and put $f(X)=\prod_{i=1}^{h(\mathcal{O})}\left(X-t\left(\tau_{\mathcal{Q}_{i}}\right)\right)$. Then $f(X)$ is the minimal polynomial of $t(\alpha)$ over $K$ and lies in $\mathbb{Z}[X]$.

Pr o of. We first construct a bijection between $\mathcal{Q}_{d_{K}}^{(2)} / \Gamma_{0}(12)$ and $\mathcal{Q}_{d_{K}}(6) /$ $\Gamma_{0}(6)$. Define $\phi: \mathcal{Q}_{d_{K}}^{(2)} / \Gamma_{0}(12) \rightarrow \mathcal{Q}_{d_{K}}(6) / \Gamma_{0}(6)$ by sending a class of $\left[a^{\prime}, b^{\prime}, c^{\prime}\right]$ to that of $\left[a^{\prime} / 2, b^{\prime}, 2 c^{\prime}\right]$. Observe that $\phi$ sends the class of $\left[a^{\prime}, b^{\prime}, c^{\prime}\right] \circ$ $\left(\begin{array}{ll}p & q \\ r & s\end{array}\right)$ (with $\left.\left(\begin{array}{ll}p & q \\ r & s\end{array}\right) \in \Gamma_{0}(12)\right)$ to the class of $\left[a^{\prime} / 2, b^{\prime}, 2 c^{\prime}\right] \circ\left(\begin{array}{cc}p & 2 q \\ r / 2 & s\end{array}\right)$, where $\left(\begin{array}{cc}p & 2 q \\ r / 2 & s\end{array}\right)$ lies in $\Gamma_{0}(6)$. Thus $\phi$ is a well defined map. Conversely, we define a map $\psi: \mathcal{Q}_{d_{K}}(6) / \Gamma_{0}(6) \rightarrow \mathcal{Q}_{d_{K}}^{(2)} / \Gamma_{0}(12)$ as follows: we observe that any class in $\mathcal{Q}_{d_{K}}(6) / \Gamma_{0}(6)$ contains a form $\left[a^{\prime \prime}, b^{\prime \prime}, c^{\prime \prime}\right]$ with $c^{\prime \prime}$ even. In fact, if $\left[a^{\prime \prime}, b^{\prime \prime}, c^{\prime \prime}\right]$ is a form in $\mathcal{Q}_{d_{K}}(6)$ with $c^{\prime \prime}$ odd, then we consider $\left[a^{\prime \prime}, b^{\prime \prime}, c^{\prime \prime}\right] \circ\left(\begin{array}{ll}7 & 1 \\ 6 & 1\end{array}\right)=\left[*, *, a^{\prime \prime}+b^{\prime \prime}+c^{\prime \prime}\right]$. Since $d_{K}=b^{\prime \prime 2}-4 a^{\prime \prime} c^{\prime \prime} \equiv 0$ 
$(\bmod 4), b^{\prime \prime}$ must be even. The fact that both $a^{\prime \prime}$ and $c^{\prime \prime}$ are odd implies that $a^{\prime \prime}+b^{\prime \prime}+c^{\prime \prime}$ is even, as desired. For such a $\left[a^{\prime \prime}, b^{\prime \prime}, c^{\prime \prime}\right]$, we define $\psi\left(\left[a^{\prime \prime}, b^{\prime \prime}, c^{\prime \prime}\right]\right)=\left[2 a^{\prime \prime}, b^{\prime \prime}, c^{\prime \prime} / 2\right]$. For $\left(\begin{array}{cc}u & v \\ w & x\end{array}\right) \in \Gamma_{0}(6)$, let $\left[a^{\prime \prime}, b^{\prime \prime}, c^{\prime \prime}\right] \circ\left(\begin{array}{ll}u & v \\ w & x\end{array}\right)=$ $\left[*, *, a^{\prime \prime} v^{2}+b^{\prime \prime} v x+c^{\prime \prime} x^{2}\right]$ have $a^{\prime \prime} v^{2}+b^{\prime \prime} v x+c^{\prime \prime} x^{2}$ even. Then the fact that $a^{\prime \prime}$ is odd and $b^{\prime \prime}, c^{\prime \prime}$ are even implies that $v$ should be even. Now $\psi$ maps $\left[a^{\prime \prime}, b^{\prime \prime}, c^{\prime \prime}\right] \circ\left(\begin{array}{ll}u & v \\ w & x\end{array}\right)$ to $\left[2 a^{\prime \prime}, b^{\prime \prime}, c^{\prime \prime} / 2\right] \circ\left(\begin{array}{cc}u & v / 2 \\ 2 w & x\end{array}\right)$, where $\left(\begin{array}{cc}u & v / 2 \\ 2 w & x\end{array}\right) \in \Gamma_{0}(12)$. Hence $\psi$ is also well defined. Further, $\phi$ and $\psi$ are inverses of each other by construction. Thus

$$
\left|\mathcal{Q}_{d_{K}}^{(2)} / \Gamma_{0}(12)\right|=\left|\mathcal{Q}_{d_{K}}(6) / \Gamma_{0}(6)\right|=h\left(\mathbb{Z}+6 \mathcal{O}_{K}\right)=h\left(\mathbb{Z}+12 \mathcal{O}_{K}\right) / 2 .
$$

Now let $\pi: \mathcal{Q}_{d_{K}}^{(2)} / \Gamma_{1}(12) \rightarrow \mathcal{Q}_{d_{K}}^{(2)} / \Gamma_{0}(12)$ be the natural projection. Then it can be easily seen that $\left|\pi^{-1}(\mathcal{Q})\right|=2$ for each $\mathcal{Q} \in \mathcal{Q}_{d_{K}}^{(2)} / \Gamma_{0}(12)$. This proves the first assertion.

For the second, we see that $f(X)$ has $t(\alpha)$ as a root due to the conditions on $a, b, c$ and $d_{K}$. If we proceed in a similar manner as in Theorem 25(2), it can be shown that the conjugates of $t(\alpha)$ over $K$ must have the form $t\left(\tau^{\prime}\right)$ with $\tau^{\prime}$ being a root of $\left[a^{\prime}, b^{\prime}, c^{\prime}\right] \in \mathcal{Q}_{d_{K}}^{(2)}$. Thus $t\left(\tau^{\prime}\right)=t\left(\tau_{\mathcal{Q}_{j}}\right)$ for some $j$. At this stage, we need to know the field degree of $K(t(\alpha))$ over $K$. By [1], Theorem 3.7.5(i), $K\left(T_{12 I}(\alpha)\right)$ is the ring class field of order $\mathbb{Z}+6 \mathcal{O}_{K}$. Since $[K(t(\alpha)): K]=2 h\left(\mathbb{Z}+6 \mathcal{O}_{K}\right)=h\left(\mathbb{Z}+12 \mathcal{O}_{K}\right)$, each $t\left(\tau_{\mathcal{Q}_{j}}\right)$ gives rise to all the conjugates of $t(\alpha)$. Finally, the proof of the fact that $f(X) \in \mathbb{Z}[X]$ is completely the same as that in Theorem 25(2).

Examples. (1) Take $K=\mathbb{Q}(\sqrt{-1})$ and $\mathfrak{a}=[2,1+\sqrt{-1}]$. Then the degree of $K\left(j_{1,12}((1+\sqrt{-1}) / 2)\right)$ over $K$ is $h\left(\mathbb{Z}+12 \mathcal{O}_{K}\right)=8$. Observe that

$$
\mathcal{Q}_{d_{K}}^{(2)} / \Gamma_{0}(12)=\{[2,-2,1],[26,10,1],[10,14,5],[10,-14,5]\} .
$$

Taking the representatives of $\mathcal{Q}_{d_{K}}^{(2)} / \Gamma_{0}(12)$ in the above and $\gamma=\left(\begin{array}{cc}7 & 4 \\ 12 & 7\end{array}\right)$ in $\Gamma_{0}(12) \backslash \pm \Gamma_{1}(12)$, we come up with the following minimal polynomial of $t((1+\sqrt{-1}) / 2)$

$$
X^{8}+28 X^{7}+124 X^{6}+304 X^{5}+448 X^{4}+340 X^{3}+208 X^{2}+64 X+16 .
$$

(2) Take $K=\mathbb{Q}(\sqrt{-2})$ and $\mathfrak{a}=[2, \sqrt{-2}]$. Then the degree of $j_{1,12}(\sqrt{-2} / 2)$ over $K$ is $h\left(\mathbb{Z}+12 \mathcal{O}_{K}\right)=8$. Observe that

$$
\mathcal{Q}_{d_{K}}^{(2)} / \Gamma_{0}(12)=\{[2,0,1],[22,-28,9],[86,32,3],[134,40,3]\} .
$$

Taking the representatives of $\mathcal{Q}_{d_{K}}^{(2)} / \Gamma_{0}(12)$ in the above and $\gamma=\left(\begin{array}{cc}7 & 4 \\ 12 & 7\end{array}\right)$ in $\Gamma_{0}(12) \backslash \pm \Gamma_{1}(12)$, we come up with the following minimal polynomial of $t(\sqrt{-2} / 2)$ :

$$
X^{8}-80 X^{7}-416 X^{6}-992 X^{5}-1280 X^{4}-896 X^{3}-224 X^{2}+64 X+16 .
$$

TheOREM 27. Notations being as in Theorem 26, assume that $(a, 12)=2$ and $d_{K} \equiv 1(\bmod 8)$. Then: 
(1) $\left|\mathcal{Q}_{d_{K}} / \Gamma_{1}(12)\right|=2 h(\mathcal{O})$, where $\mathcal{O}=\mathbb{Z}+12 \mathcal{O}_{K}$.

(2) $g(X):=\prod_{i=1}^{2 h(\mathcal{O})}\left(X-t\left(\tau_{\mathcal{Q}_{i}}\right)\right)$ has $t(\alpha)$ as a root and lies in $\mathbb{Z}[X]$. Let $f(X) \in K[X]$ be the monic irreducible factor of $g(X)$ having $t(\alpha)$ as a root. Then $f(X)$ is the minimal polynomial of $t(\alpha)$ over $K$ and lies in $\mathcal{O}_{K}[X]$.

Proof. (1) We define $\phi: \mathcal{Q}_{d_{K}}^{(2)} / \Gamma_{0}(12) \rightarrow \mathcal{Q}_{d_{K}}(6) / \Gamma_{0}(6,2)$ by sending the class of $\left[a^{\prime}, b^{\prime}, c^{\prime}\right]$ to that of $\left[a^{\prime} / 2, b^{\prime}, 2 c^{\prime}\right]$. Observe that $\phi$ sends the class

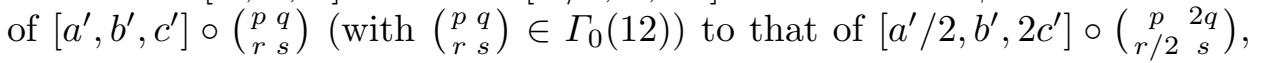
where $\left(\begin{array}{cc}p & 2 q \\ r / 2 & s\end{array}\right)$ lies in $\Gamma_{0}(6,2)$. Thus $\phi$ is a well defined map. Conversely, we define $\psi: \mathcal{Q}_{d_{K}}(6) / \Gamma_{0}(6,2) \rightarrow \mathcal{Q}_{d_{K}}^{(2)} / \Gamma_{0}(12)$ as follows: we note that, for any class $\left[a^{\prime \prime}, b^{\prime \prime}, c^{\prime \prime}\right]$ in $\mathcal{Q}_{d_{K}}(6) / \Gamma_{0}(6,2), c^{\prime \prime}$ is always even because $a^{\prime \prime}$ is odd and $d_{K}=b^{\prime \prime 2}-4 a^{\prime \prime} c^{\prime \prime} \equiv 1(\bmod 8)$. Now $\psi$ sends $\left[a^{\prime \prime}, b^{\prime \prime}, c^{\prime \prime}\right] \circ\left(\begin{array}{ll}u & v \\ w & x\end{array}\right)$ to $\left[2 a^{\prime \prime}, b^{\prime \prime}, c^{\prime \prime} / 2\right] \circ\left(\begin{array}{cc}u & v / 2 \\ 2 w & x\end{array}\right)$, where $\left(\begin{array}{cc}u & v / 2 \\ 2 w & x\end{array}\right) \in \Gamma_{0}(12)$. Hence $\psi$ is also well defined. Moreover, $\phi$ and $\psi$ are inverses of each other. Thus

$$
\left|\mathcal{Q}_{d_{K}}^{(2)} / \Gamma_{0}(12)\right|=\left|\mathcal{Q}_{d_{K}}(6) / \Gamma_{0}(6,2)\right|=2\left|\mathcal{Q}_{d_{K}}(6) / \Gamma_{0}(6)\right|=h(\mathcal{O}) .
$$

This implies that $\left|\mathcal{Q}_{d_{K}}^{(2)} / \Gamma_{1}(12)\right|=2 h(\mathcal{O})$, which proves (1).

(2) The assertion $g(t(\alpha))=0$ and $g(X) \in \mathbb{Z}[X]$ can be proved by the same method as in Theorem 26. The remaining assertions are obvious.

ExAmple. Take $K=\mathbb{Q}(\sqrt{-7})$ and $\mathfrak{a}=[2,(-1+\sqrt{-7}) / 2]$. The degree of $K\left(j_{1,12}((-1+\sqrt{-7}) / 4)\right)$ over $K$ is $h\left(\mathbb{Z}+12 \mathcal{O}_{K}\right)=8$. Observe that

$$
\begin{aligned}
\mathcal{Q}_{d_{K}}^{(2)} / \Gamma_{0}(12)=\{ & \{[2,1,1],[2,-1,1],[22,13,2],[22,-13,2], \\
& {[14,21,8],[14,-21,8],[106,29,2],[106,-29,2]\} . }
\end{aligned}
$$

Then we have an irreducible polynomial over $\mathbb{Z}$,

$$
\begin{aligned}
g(X)= & X^{16}+8 X^{15}+4104 X^{14}+32656 X^{13}+138848 X^{12}+401328 X^{11} \\
& +866800 X^{10}+1464128 X^{9}+1980720 X^{8}+2173760 X^{7} \\
& +1946944 X^{6}+1423872 X^{5}+843008 X^{4}+394240 X^{3}+138240 X^{2} \\
& +32768 X+4096,
\end{aligned}
$$

which has $t(\alpha)$ as a root. However, since the degree of $K(t((-1+\sqrt{-7}) / 4))$ over $K$ is 8 , we must factor $g(X)$ into two polynomials in $\mathcal{O}_{K}[X]$ and one of them is the minimal polynomial of $t(\alpha)$. Indeed, we come up with the following minimal polynomial of $t(\alpha)$ over $K$ :

$$
\begin{aligned}
X^{8}+ & (4-24 \sqrt{-7}) X^{7}+(28-96 \sqrt{-7}) X^{6}+(88-216 \sqrt{-7}) X^{5} \\
& +(136-312 \sqrt{-7}) X^{4}+(88-312 \sqrt{-7}) X^{3}-(8+216 \sqrt{-7}) X^{2} \\
& -(32+96 \sqrt{-7}) X-(8+24 \sqrt{-7}) .
\end{aligned}
$$

Lastly, for more practical and overall calculation of minimal polynomials, we first need the following lemma. 
Lemma 28. For each even integer $N \geq 4$, let

$\gamma_{1}=\left(\begin{array}{ll}1 & 0 \\ 0 & 1\end{array}\right), \quad \gamma_{2}=\left(\begin{array}{ll}1 & 0 \\ 1 & 1\end{array}\right), \quad \gamma_{n+1}=\left(\begin{array}{cc}n+1 & 1 \\ n & 1\end{array}\right) \quad(2 \leq n \leq N-1)$

and

$$
\delta_{m}=\left(\begin{array}{cc}
2 m+1 & 4 m+1 \\
1 & 2
\end{array}\right) \quad(1 \leq m \leq N / 2-1) .
$$

Then the set $\left\{\gamma_{1}, \ldots, \gamma_{N}, \delta_{1}, \ldots, \delta_{N / 2-1}\right\}$ is a subset of representatives for $\bar{\Gamma}(1) / \bar{\Gamma}_{0}(N)$.

Proof. First, we check that $\gamma_{i}^{-1} \gamma_{j} \notin \Gamma_{0}(N)$ for distinct $i$ and $j$. We have

$\gamma_{2}^{-1} \gamma_{n+1}=\left(\begin{array}{cc}n+1 & 1 \\ -1 & 0\end{array}\right) \notin \Gamma_{0}(N) \quad$ and $\quad \gamma_{m+1}^{-1} \gamma_{n+1}=\left(\begin{array}{cc}1 & 0 \\ n-m & 1\end{array}\right) \in \Gamma_{0}(N)$

if and only if $n=m$ because $2 \leq n, m \leq N-1$. And $\gamma_{2}^{-1} \delta_{m}=\left(\begin{array}{c}* \\ -2 m \\ -2 m\end{array}\right) \notin$ $\Gamma_{0}(N)$ since $-N+2 \leq-2 m \leq-2$, and $\delta_{m}^{-1} \delta_{n}=(\underset{2(m-n) *}{*}) \in \Gamma_{0}(N)$ if and only if $m=n$ owing to the fact that $-(N-4) \leq 2(m-n) \leq N-4$. Finally, we get $\gamma_{n+1}^{-1} \delta_{m}=\left(\begin{array}{cc}* & * \\ -2 m n+1 & *\end{array}\right) \notin \Gamma_{0}(N)$ because $-2 m n+1$ is an odd integer. This proves the lemma.

For our case $N=12$,

$$
\gamma_{1}=\left(\begin{array}{ll}
1 & 0 \\
0 & 1
\end{array}\right), \quad \gamma_{2}=\left(\begin{array}{ll}
1 & 0 \\
1 & 1
\end{array}\right), \quad \gamma_{n+1}=\left(\begin{array}{cc}
n+1 & 1 \\
n & 1
\end{array}\right) \quad(2 \leq n \leq 11)
$$

and

$$
\delta_{m}=\left(\begin{array}{cc}
2 m+1 & 4 m+1 \\
1 & 2
\end{array}\right) \quad(1 \leq m \leq 5)
$$

constitute a part of the set of representatives for $\bar{\Gamma}(1) / \bar{\Gamma}_{0}(12)$.

Then from a direct computation we can show that

$$
\begin{gathered}
\gamma_{13}=\left(\begin{array}{ll}
1 & -1 \\
3 & -2
\end{array}\right), \quad \gamma_{14}=\left(\begin{array}{ll}
7 & 2 \\
3 & 1
\end{array}\right), \quad \gamma_{15}=\left(\begin{array}{ll}
1 & 1 \\
4 & 5
\end{array}\right), \quad \gamma_{16}=\left(\begin{array}{ll}
2 & -1 \\
5 & -2
\end{array}\right), \\
\gamma_{17}=\left(\begin{array}{ll}
4 & 1 \\
7 & 2
\end{array}\right), \quad \gamma_{18}=\left(\begin{array}{cc}
1 & 1 \\
10 & 11
\end{array}\right), \quad \gamma_{19}=\left(\begin{array}{cc}
2 & -1 \\
1 & 0
\end{array}\right)
\end{gathered}
$$

together with $\left\{\gamma_{1}, \ldots, \gamma_{12}, \delta_{1}, \ldots, \delta_{5}\right\}$ form a complete set of representatives for $\bar{\Gamma}(1) / \bar{\Gamma}_{0}(12)$. Define $S=\left\{\gamma_{1}, \ldots, \gamma_{19}, \delta_{1}, \ldots, \delta_{5}\right\}$. Since $\left(\begin{array}{cc}7 & 4 \\ 12 & 7\end{array}\right) \in$ $\Gamma_{0}(12) \backslash \pm \Gamma_{1}(12)$, we see that $S^{\prime}=S \cup S\left(\begin{array}{cc}7 & 4 \\ 12 & 7\end{array}\right)$ is a complete set of representatives for $\bar{\Gamma}(1) / \bar{\Gamma}_{1}(12)$ as desired.

TheOREM 29. With $K$ and $\alpha$ as before, let $f(X)$ be the minimal polynomial of $t(\alpha)$ over $K$ and $a z^{2}+b z+c=0$ the equation of $\alpha$ such that $a>0$ 
and $(a, b, c)=1$. Let $\mathcal{Q}_{d_{K}} / \Gamma(1)=\left\{\mathcal{Q}_{j}\right\}_{j=1}^{h_{K}}$ and $\bar{\Gamma}(1) / \bar{\Gamma}_{1}(12)=\left\{\gamma_{k}\right\}_{k=1}^{48}$ with $\gamma_{k} \in S^{\prime}$, where $h_{K}$ denotes the class number of $K$. Define

$$
g(X)=\prod_{j=1}^{h_{K}} \prod_{k=1}^{48}\left(X-t\left(\gamma_{k}^{-1} \tau_{\mathcal{Q}_{j}}\right)\right) .
$$

Then:

(1) $g(X)$ lies in $\mathbb{Z}[X]$ and is divisible by $f(X)$.

(2) $f(X)$ lies in $\mathcal{O}_{K}[X] \backslash \mathbb{R}[X]$ if

$$
\left\{\begin{array}{l}
(a, 12)=2,4,12 \text { and } d_{K} \equiv 1(\bmod 8) \\
(a, 12)=3 \text { and } b \not \equiv 0(\bmod 3), \\
(a, 12)=6 \text { and } b \neq \equiv 0(\bmod 6)
\end{array}\right.
$$

and lies in $\mathbb{Z}[X]$ if

$$
\left\{\begin{array}{l}
(a, 12)=1 \\
(a, 12)=2 \text { and } d_{K} \equiv 0(\bmod 4), \\
(a, 12)=3 \text { and } b \equiv 0(\bmod 3), \\
(a, 12)=6 \text { and } b \equiv 0(\bmod 6) .
\end{array}\right.
$$

(3) $g(X)$ decomposes in the following way:

$$
\begin{cases}f_{1}(X)^{3} f_{3}(X)^{3} & \text { if } d_{K}=-3, \\ f_{1}(X)^{2} f_{2}(X)^{2} & \text { if } d_{K}=-4, \\ f_{1}(X)^{n_{1}}\left(f_{2}(X) \overline{f_{2}(X)}\right)^{n_{2}}\left(f_{3}(X) \overline{f_{3}(X)}\right)^{n_{3}}\left(f_{4}(X) \overline{f_{4}(X)}\right)^{n_{4}} & \\ \times\left(f_{6}(X) \overline{f_{6}(X)}\right)^{n_{6}}\left(f_{12}(X) \overline{f_{12}(X)}\right)^{n_{12}} & \text { if } d_{K} \equiv 1(\bmod 8), d_{K} \equiv \pm 1(\bmod 12), \\ f_{1}(X) f_{2}(X) \overline{f_{2}(X)} f_{4}(X) \overline{f_{4}(X)} & \text { if } d_{K} \equiv 1(\bmod 8), d_{K} \equiv \pm 5(\bmod 12), \\ f_{1}(X) f_{2}(X) \overline{f_{2}(X)} f_{3}(X) f_{4}(X) \overline{f_{4}(X)} f_{6}(X) \overline{f_{6}(X)} f_{12}(X) \overline{f_{12}(X)} & \text { if } d_{K} \equiv 1(\bmod 8), d_{K} \equiv 0(\bmod 3), \\ f_{1}(X) f_{3}(X) \overline{f_{3}(X)} & \text { if } d_{K} \equiv 5(\bmod 8), d_{K} \equiv \pm 1(\bmod 12), \\ f_{1}(X) & \text { if } d_{K} \equiv 5(\bmod 8), d_{K} \equiv \pm 5(\bmod 12), \\ f_{1}(X) f_{3}(X) & \text { if } d_{K} \equiv 5(\bmod 8), d_{K} \equiv 0(\bmod 3), \\ f_{1}(X) f_{2}(X) f_{3}(X) f_{6}(X) & \text { if } d_{K} \equiv 0(\bmod 4), d_{K} \equiv 0(\bmod 3), \\ f_{1}(X) f_{2}(X) f_{3}(X) \overline{f_{3}(X)} f_{6}(X) \overline{f_{6}(X)} & \text { if } d_{K} \equiv 0(\bmod 4), d_{K} \equiv 1(\bmod 3), \\ f_{1}(X) f_{2}(X) & \text { if } d_{K} \equiv 0(\bmod 4), d_{K} \equiv 2(\bmod 3),\end{cases}
$$


where $f_{i}(X)(i=1,2,3,4,6,12)$ stands for the minimal polynomial of $t(\alpha)$ over $K$ with $(a, 12)=i$, and $\overline{f_{i}(X)}$ the complex conjugation of $f_{i}(X)$. In the third case, each $n_{j} \geq 1$ and

$$
8\left(n_{1}+n_{2}+n_{3}+n_{4}\right)+4\left(n_{6}+n_{12}\right)=48 .
$$

Proof. (1) Let $\pi: \mathcal{Q}_{d_{K}} / \Gamma_{1}(12) \rightarrow \mathcal{Q}_{d_{K}} / \Gamma(1)$ be the natural projection. Then for each $\mathcal{Q}_{j} \in \mathcal{Q}_{d_{K}} / \Gamma(1), \pi^{-1}\left(\mathcal{Q}_{j}\right)=\left\{\mathcal{Q}_{j} \circ \gamma_{k} \mid k=1, \ldots, 48\right\}$. Hence, $[a, b, c]$ is equivalent under $\Gamma_{1}(12)$ to $\mathcal{Q}_{j} \circ \gamma_{k}$ for some $j$ and $k$ because $[a, b, c]$ belongs to $\mathcal{Q}_{d_{K}}$. Since $t(\alpha)=t\left(\gamma_{k}^{-1} \tau_{\mathcal{Q}_{j}}\right), g(X)$ certainly has $t(\alpha)$ as a root. Moreover, the fact that $g(X) \in \mathbb{Z}[X]$ can be proved in the same manner as in Theorem 25(2).

(2) Let $\tau$ be the map which gives the complex conjugation on $K(t(\alpha))$. Then it can be easily shown that

$$
\operatorname{Ker}\left(\Phi_{K(t(\alpha))^{\tau} / K}\right)=\left(\operatorname{Ker}\left(\Phi_{K(t(\alpha)) / K}\right)\right)^{\tau}=P_{K, 1}(\mathfrak{f})^{\tau}
$$

where $\mathfrak{f}$ is as in Table 1 .

If $(a, 12) \geq 2$ and the conditions in the first statement are satisfied, then we can see from the proof of Theorem 21 that either 2 or 3 splits completely in $K$, and so $P_{K, 1}(\mathfrak{f})^{\tau}=P_{K, 1}\left(\mathfrak{f}^{\tau}\right) \neq P_{K, 1}(\mathfrak{f})$. This implies that $K(t(\alpha))^{\tau} \neq K(t(\alpha))$. Moreover, $f(X)$ differs from $\overline{f(X)}$ because $K(t(\alpha))$ (resp. $\left.K(t(\alpha))^{\tau}\right)$ is the splitting field of $f(X)$ (resp. $\overline{f(X)}$ ). Therefore we conclude that $f(X) \notin \mathbb{R}[X]$.

For the cases $(a, 12)=1,(a, 12)=2$ and $d_{K} \equiv 0(\bmod 4)$, the assertion follows from Theorems 25 and 26 (this can also be proved by the argument below). For the other cases, we note that the conductors $\mathfrak{f}$ are of the form "an integer times a product of ramified prime ideals". Therefore, $\mathfrak{f}$ should be invariant under the action of $\tau$ and so

$$
\begin{aligned}
\operatorname{Gal}(K(t(\alpha)) / K) & \cong I_{K}(\mathfrak{f}) / P_{K, 1}(\mathfrak{f})=I_{K}\left(\mathfrak{f}^{\tau}\right) / P_{K, 1}\left(\mathfrak{f}^{\tau}\right) \\
& \cong \operatorname{Gal}\left(K(t(\alpha))^{\tau} / K\right) .
\end{aligned}
$$

Hence, it follows from the uniqueness theorem of class field theory that

$$
K(t(\alpha))=K(t(\alpha))^{\tau}=K\left(t(\alpha)^{\tau}\right) .
$$

Then, since both $K(t(\alpha))$ and $K\left(t(\alpha)^{\tau}\right)$ are splitting fields of $f(X)$, they are identical. This yields that

$$
f(X)=\overline{f(X)} \quad \text { and } \quad f(X) \in\left(\mathcal{O}_{K} \cap \mathbb{R}\right)[X]=\mathbb{Z}[X] .
$$

(3) If $d_{K}=-3$ (resp. $d_{K}=-4$ ), the decomposition of $g(X)$ is immediately obtained by factorizing the polynomial $\prod_{k=1}^{48}\left(X-t\left(\gamma_{k}^{-1} \varrho\right)\right)$ (resp. $\left.\prod_{k=1}^{48}\left(X-t\left(\gamma_{k}^{-1} \sqrt{-1}\right)\right)\right)$ where $\varrho=e^{2 \pi i / 3}$. Next, suppose that $d_{K} \neq-3,-4$. 
Let $\mathfrak{f}$ be as in Theorem 21 . We then see that

$$
\begin{aligned}
{\left[K_{\mathfrak{f}}: K\right] } & =\left[K_{\mathfrak{f}}: K\left(j_{0,12}(\alpha)\right)\right]\left[K\left(j_{0,12}(\alpha)\right): K\right]=2\left[K\left(j_{0,12}(\alpha)\right): K\right] \\
& =2 h\left(\mathcal{O}_{f}\right) \quad \text { by }[1], \text { Theorem 3.7.5(i), }
\end{aligned}
$$

for an imaginary quadratic order $\mathcal{O}_{f}=\mathbb{Z}+f \mathcal{O}_{K}$ where $f=12 /(a, 12)$. As for the computation of $h\left(\mathcal{O}_{f}\right)$, we recall from [16] or [19] that

$$
h\left(\mathcal{O}_{f}\right)=h_{K} \frac{f}{\left(\mathcal{O}_{K}^{\times}: \mathcal{O}_{f}^{\times}\right)} \prod_{p \mid f}\left(1-\left(\frac{d_{K}}{p}\right) \frac{1}{p}\right),
$$

where $h_{K}$ is the class number of $K, \mathcal{O}_{K}^{\times}$and $\mathcal{O}_{f}^{\times}$are the unit groups of $\mathcal{O}_{K}$ and $\mathcal{O}_{f}$, respectively, and $\left(\frac{d_{K}}{p}\right)$ is the quadratic reciprocity, equal to 1 if $p$ splits completely in $K,-1$ if $p$ inerts, and 0 if $p$ ramifies in $K$. By the assertion (1), the polynomials on the right hand side are factors of $g(X)$. Furthermore, we see by (7) that the sum of their degrees in each case is equal to the degree of $g(X)$, which is $48 h_{K}$. This completes the proof.

Given $K$ and $\alpha$, factorizing the polynomial $g(X)$ in Theorem 29, we

\begin{tabular}{|c|c|c|c|}
\hline \multicolumn{4}{|c|}{$d_{K}=-3$} \\
\hline$\alpha$ & $(a, 12)$ & $\mathfrak{f}$ & $\min (t(\alpha), K)$ \\
\hline$\frac{-1+\sqrt{-3}}{2}$ & $\underline{3}$ & (12) & $\begin{array}{l}X^{12}+240 X^{11}+2172 X^{10}+9752 X^{9} \\
\quad+27324 X^{8}+52416 X^{7}+71520 X^{6} \\
\quad+69696 X^{5}+47088 X^{4}+20480 X^{3} \\
+4800 X^{2}+384 X+64\end{array}$ \\
\hline$\frac{-3+\sqrt{-3}}{6}$ & 3 & $4\left[3, \frac{-3+\sqrt{-3}}{2}\right]$ & $X^{4}+8 X^{3}+12 X^{12}+8 X+4$ \\
\hline \multicolumn{4}{|c|}{$d_{K}=-4$} \\
\hline$\alpha$ & $(a, 12)$ & $\mathfrak{f}$ & $\min (t(\alpha), K)$ \\
\hline$\sqrt{-1}$ & 1 & (12) & $\begin{array}{l}X^{16}-520 X^{15}-8184 X^{14}-59840 X^{13} \\
-266800 X^{12}-813984 X^{11} \\
-1810976 X^{10}-3051904 X^{9} \\
-3978144 X^{8}-4039552 X^{7} \\
-317504 X^{6}-1886208 X^{5} \\
-803584 X^{4}-218624 X^{3} \\
-26112 X^{2}+2048 X+256\end{array}$ \\
\hline$\frac{1+\sqrt{-1}}{2}$ & 2 & {$[2,1+\sqrt{-1}]^{3}$} & $\begin{array}{l}X^{8}+28 X^{7}+124 X^{6}+304 X^{5}+448 X^{4} \\
\quad+340 X^{3}+208 X^{2}+64 X+16\end{array}$ \\
\hline
\end{tabular}
obtain the following table for several $d_{K} \geq-7$.

Table 2. Minimal polynomial of $t(\alpha)$ 
Table 2 (cont.)

$d_{K}=-7$

\begin{tabular}{|c|c|c|c|}
\hline$\alpha$ & $(a, 12)$ & $\mathfrak{f}$ & $\min (t(\alpha), K)$ \\
\hline$\frac{-1+\sqrt{-7}}{2}$ & 1 & $(12)$ & $\begin{array}{l}X^{16}+4088 X^{15}+65544 X^{14} \\
+479296 X^{13}+2133968 X^{12} \\
+6508128 X^{11}+14487520 X^{10} \\
+24430208 X^{9}+31839840 X^{8} \\
+32289920 X^{7}+25339264 X^{6} \\
+15071232 X^{5}+6495488 X^{4} \\
+1845760 X^{3}+268800 X^{2} \\
+2048 X+256 \\
\end{array}$ \\
\hline$\frac{-1+\sqrt{-7}}{4}$ & 2 & $\begin{array}{l}3\left[2, \frac{-1+\sqrt{-7}}{2}\right] \\
\times\left[2, \frac{1+\sqrt{-7}}{2}\right]^{2}\end{array}$ & $\begin{array}{l}X^{8}+(4-24 \sqrt{-7}) X^{7}+(28-96 \sqrt{-7}) X^{6} \\
+(88-216 \sqrt{-7}) X^{5}+(136-312 \sqrt{-7}) X^{4} \\
+(88-312 \sqrt{-7}) X^{3}-(8+216 \sqrt{-7}) X^{2} \\
-(32+96 \sqrt{-7}) X-(8+24 \sqrt{-7})\end{array}$ \\
\hline$\frac{1+\sqrt{-7}}{4}$ & 2 & $\begin{aligned} & 3\left[2, \frac{1+\sqrt{-7}}{2}\right] \\
\times & {\left[2, \frac{-1+\sqrt{-7}}{2}\right]^{2} }\end{aligned}$ & $\begin{array}{l}X^{8}+(4+24 \sqrt{-7}) X^{7}+(28+96 \sqrt{-7}) X^{6} \\
+(88+216 \sqrt{-7}) X^{5}+(136+312 \sqrt{-7}) X^{4} \\
+(88+312 \sqrt{-7}) X^{3}-(8-216 \sqrt{-7}) X^{2} \\
-(32-96 \sqrt{-7}) X-(8-24 \sqrt{-7})\end{array}$ \\
\hline$\frac{-3+\sqrt{-7}}{8}$ & 4 & $3\left[2, \frac{-1+\sqrt{-7}}{2}\right]^{2}$ & $\begin{array}{l}X^{8}+\left(\frac{23-3 \sqrt{-7}}{2}\right) X^{7}+(58-6 \sqrt{-7}) X^{6} \\
+\left(\frac{311-27 \sqrt{-7}}{2}\right) X^{5}+\left(\frac{467-39 \sqrt{-7}}{2}\right) X^{4} \\
+\left(\frac{371-39 \sqrt{-7}}{2}\right) X^{3}+\left(\frac{119-27 \sqrt{-7}}{2}\right) X^{2} \\
-(2+6 \sqrt{-7}) X-\left(\frac{1+3 \sqrt{-7}}{2}\right)\end{array}$ \\
\hline$\frac{3+\sqrt{-7}}{8}$ & 4 & $3\left[2, \frac{1+\sqrt{-7}}{2}\right]^{2}$ & $\begin{array}{l}X^{8}+\left(\frac{23+3 \sqrt{-7}}{2}\right) X^{7}+(58+6 \sqrt{-7}) X^{6} \\
+\left(\frac{311+27 \sqrt{-7}}{2}\right) X^{5}+\left(\frac{467+39 \sqrt{-7}}{2}\right) X^{4} \\
+\left(\frac{371+39 \sqrt{-7}}{2}\right) X^{3}+\left(\frac{119+27 \sqrt{-7}}{2}\right) X^{2} \\
-(2-6 \sqrt{-7}) X-\left(\frac{1-3 \sqrt{-7}}{2}\right)\end{array}$ \\
\hline
\end{tabular}

Here $\min (t(\alpha), K)$ denotes the minimal polynomial of $t(\alpha)$ over $K$.

Appendix. In Table 3, we give the Hauptmoduln for the genus zero curves $X_{0}(N)$, due to K. Harada $([4])$. Note that each Hauptmodul corresponds to the Thompson series as specified in the table ([2]).

For generation of generators of $K\left(X_{1}(N)\right)$, we used the functions:

- $E_{4}(z)=1+240 \sum_{n=1}^{\infty} \sigma_{3}(n) q^{n}$, the normalized Eisenstein series of weight 4 , 
Table 3

\begin{tabular}{|l|l|c|}
\hline$N$ & \multicolumn{1}{|c|}{ Hauptmodul } & Type \\
\hline 2 & $\frac{\eta(z)^{24}}{\eta(2 z)^{24}}$ & $2 \mathrm{~B}$ \\
\hline 3 & $\frac{\eta(z)^{12}}{\eta(3 z)^{12}}$ & $3 \mathrm{~B}$ \\
\hline 4 & $\frac{\eta(z)^{8}}{\eta(4 z)^{8}}, \frac{\eta(2 z)^{24}}{\eta(z)^{8} \eta(4 z)^{16}}$ & $4 \mathrm{C}$ \\
\hline 5 & $\frac{\eta(z)^{6}}{\eta(5 z)^{6}}$ & $5 \mathrm{~B}$ \\
\hline 6 & $\frac{\eta(2 z)^{3} \eta(3 z)^{9}}{\eta(z)^{3} \eta(6 z)^{9}}, \frac{\eta(2 z)^{8} \eta(3 z)^{4}}{\eta(z)^{4} \eta(6 z)^{8}}, \frac{\eta(z)^{5} \eta(3 z)}{\eta(2 z) \eta(6 z)^{5}}$ & $6 \mathrm{E}$ \\
\hline 7 & $\frac{\eta(z)^{4}}{\eta(7 z)^{4}}$ & $7 \mathrm{~B}$ \\
\hline 8 & $\frac{\eta(z)^{4} \eta(4 z)^{2}}{\eta(2 z)^{2} \eta(8 z)^{4}}$ & $8 \mathrm{E}$ \\
\hline 9 & $\frac{\eta(z)^{3}}{\eta(9 z)^{3}}$ & $9 \mathrm{~B}$ \\
\hline 10 & $\frac{\eta(2 z) \eta(5 z)^{5}}{\eta(z) \eta(10 z)^{5}}, \frac{\eta(2 z)^{4} \eta(5 z)^{2}}{\eta(z)^{2} \eta(10 z)^{4}}, \frac{\eta(z){ }^{3} \eta(5 z)}{\eta(2 z) \eta(10 z)^{3}}$ & $10 \mathrm{E}$ \\
\hline 12 & $\frac{\eta(4 z)^{4} \eta(6 z)^{2}}{\eta(2 z)^{2} \eta(12 z)^{4}}, \frac{\eta(3 z)^{3} \eta(4 z)}{\eta(z) \eta(12 z)^{3}}, \frac{\eta(z)^{3} \eta(4 z) \eta(6 z)^{2}}{\eta(2 z)^{2} \eta(3 z) \eta(12 z)^{3}}$ & $12 \mathrm{I}$ \\
\hline 13 & $\frac{\eta(z)^{2}}{\eta(13 z)^{2}}$ & $13 \mathrm{~B}$ \\
\hline 16 & $\frac{\eta(z)^{2} \eta(8 z)}{\eta(2 z) \eta(16 z)^{2}}$ & $16 \mathrm{~B}$ \\
\hline 18 & $\frac{\eta(6 z) \eta(9 z)^{3}}{\eta(3 z) \eta(18 z)^{3}}, \frac{\eta(2 z)^{2} \eta(9 z)}{\eta(z) \eta(18 z)^{2}}, \frac{\eta(z)^{2} \eta(6 z) \eta(9 z)}{\eta(2 z) \eta(3 z) \eta(18 z)^{2}}$ & $18 \mathrm{D}$ \\
\hline 25 & $\frac{\eta(z)}{\eta(25 z)}$ & $25 \mathrm{Z}$ \\
\hline
\end{tabular}

- $\eta(z)=e^{\pi i z / 12} \prod_{n=1}^{\infty}\left(1-q^{n}\right)$, the Dedekind eta function,

- $G_{2}(z)=2 \zeta(2)-8 \pi^{2} \sum_{n=1}^{\infty} \sigma_{1}(n) q^{n}$, the Eisenstein series of weight 2,

- $E_{2}(z)$, the normalized Eisenstein series of weight 2,

- $G_{2}^{(p)}(z)=G_{2}(z)-p G_{2}(p z)$ for a prime $p$,

- $E_{2}^{(p)}(z)=E_{2}(z)-p E_{2}(p z)$ for a prime $p$

- $G_{2}^{\left(a_{1}, a_{2}\right)(\bmod N)}(z)$, the level $N$ Eisenstein series of weight 2.

In Table 4, we give the Hauptmoduln for genus zero curves $X_{1}(N)$, due to Kim and Koo ([5]-[11]).

Since

$$
\pm\left(\begin{array}{ll}
3 & 0 \\
0 & 1
\end{array}\right)^{-1} \Gamma(3)\left(\begin{array}{ll}
3 & 0 \\
0 & 1
\end{array}\right)=\Gamma_{0}(9)
$$

and $\eta(z)^{3} / \eta(9 z)^{3}$ is the Hauptmodul of $X_{0}(9)$, we see that $j_{3}(z)$ defined above is the Hauptmodul of $X(3)$. Here, $W_{N}=\left(\begin{array}{cc}0 & -1 \\ N & 0\end{array}\right)$ is the Fricke involution. 
Table 4

\begin{tabular}{|l|l|l|}
\hline$N$ & \multicolumn{1}{|c|}{ Hauptmodul } & \multicolumn{1}{|c|}{ Field generator } \\
\hline 2 & $N\left(j_{2}(z)\right)=\frac{16}{j_{2}(z)}-8$ & $j_{2}(z)=\lambda(z)=\frac{\theta_{2}(z)^{4}}{\theta_{3}(z)^{4}}$ \\
\hline 3 & $N\left(j_{3}(z)\right)=j_{3}(z)$ & $\left.j_{3}(z)=\frac{\eta(z)^{3}}{\eta(9 z)^{3}} \mid \begin{array}{c}\frac{1}{3} 0 \\
0\end{array}\right)$ \\
\hline 4 & $N\left(j_{4}(z)\right)=\frac{4}{j_{4}(z)}+2$ & $j_{4}(z)=\frac{\theta_{3}(z / 2)}{\theta_{4}(z / 2)}$ \\
\hline \hline 2 & $N\left(j_{1,2}(z)\right)=\frac{2^{8}}{j_{1,2}(z)}+24$ & $j_{1,2}(z)=\frac{\theta_{2}(z)^{8}}{\theta_{4}(2 z)^{8}}$ \\
\hline 3 & $N\left(j_{1,3}(z)\right)=\frac{240}{j_{1,3}(z)-1}+9$ & $j_{1,3}(z)=\frac{E_{4}(z)}{E_{4}(3 z)}$ \\
\hline 4 & $N\left(j_{1,4}(z)\right)=\frac{16}{j_{1,4}(z)}-8$ & $j_{1,4}(z)=\frac{\theta_{2}(2 z)^{4}}{\theta_{3}(2 z)^{4}}$ \\
\hline 5 & $N\left(j_{1,5}(z)\right)=\frac{-8}{j_{1,5}(z)+44}-5$ & $j_{1,5}(z)=\left(4 \frac{\eta(z)^{5}}{\eta(5 z)}+E_{2}^{(5)}(z)\right) / \frac{\eta(5 z)^{5}}{\eta(z)}$ \\
\hline 6 & $N\left(j_{1,6}(z)\right)=\frac{2}{j_{1,6}(z)-1}-1$ & $j_{1,6}(z)=\frac{G_{2}^{(2)}(z)-G_{2}^{(2)}(3 z)}{2 G_{2}^{(2)}(z)-G_{2}^{(3)}(z)}$ \\
\hline 7 & $N\left(j_{1,7}(z)\right)=\frac{-1}{W_{7}\left(j_{1,7}(z)\right)-1}-3$ & $j_{1,7}(z)=\frac{G_{2}^{(0,1)(\bmod 7)}-G_{2}^{(0,2)(\bmod 7)}}{G_{2}^{(0,1)(\bmod 7)}-G_{2}^{(0,3)(\bmod 7)}}$ \\
\hline 8 & $N\left(j_{1,8}(z)\right)=\frac{2}{j_{1,8}(z)-1}-1$ & $j_{1,8}(z)=\frac{\theta_{3}(2 z)}{\theta_{3}(4 z)}$ \\
\hline 9 & $N\left(j_{1,9}(z)\right)=\frac{-1}{W_{9}\left(j_{1,9}(z)\right)-1}-2$ & $j_{1,9}(z)=\frac{G_{2}^{(0,1)(\bmod 9)}-G_{2}^{(0,2)(\bmod 9)}}{G_{2}^{(0,1)(\bmod 9)}-G_{2}^{(0,4)(\bmod 9)}}$ \\
\hline 10 & $N\left(j_{1,10}(z)\right)=\frac{-1}{W_{10}\left(j_{1,10}(z)\right)-1}-2$ & $j_{1,10}(z)=\frac{G_{2}^{(0,1)(\bmod 10)}-G_{2}^{(0,2)(\bmod 10)}}{G_{2}^{(0,1)(\bmod 10)}-G_{2}^{(0,4)(\bmod 10)}}$ \\
\hline 12 & $N\left(j_{1,12}(z)\right)=\frac{2}{j_{1,12}(z)-1}$ & $j_{1,12}(z)=\frac{\theta_{3}(2 z)}{\theta_{3}(6 z)}$ \\
\hline
\end{tabular}

\section{References}

[1] I. Chen and N. Yui, Singular values of Thompson series, in: Groups, Difference Sets and the Monster, K. T. Arasu et al. (eds.), de Gruyter, 1996, 255-326.

[2] J. H. Conway and S. P. Norton, Monstrous Moonshine, Bull. London Math. Soc. 11 (1979), 308-339.

[3] D. A. Cox, Primes of the Form $x^{2}+n y^{2}$, Wiley, 1989.

[4] K. Harada, Moonshine of Finite Groups, lecture note, Ohio State Univ.

[5] C. H. Kim and J. K. Koo, Arithmetic of the modular function $j_{4}$, J. Korean Math. Soc. 36 (1999), 707-724.

[6] - - - Arithmetic of the modular function $j_{1,4}$, Acta Arith. 84 (1998), 129-143.

[7] - - - Arithmetic of the modular function $j_{1,8}$, Ramanujan J., to appear.

[8] — - Arithmetic of the modular functions $j_{1,5}$ and $j_{1,6}$, in preparation.

[9] -, 一, Arithmetic of the modular functions $j_{1,2}$ and $j_{1,3}$, in preparation.

[10] - - Generation of Hauptmoduln of $\Gamma_{1}(7), \Gamma_{1}(9)$ and $\Gamma_{1}(10)$, in preparation.

[11] - - - On the Hauptmodul of $\Gamma_{1}(12)$, preprint. 
[12] C. H. Kim and J. K. Koo, On the genus of some modular curve of level N, Bull. Austral. Math. Soc. 54 (1996), 291-297.

[13] - - - The normalizer of $\Gamma_{1}(N)$ in $P S L_{2}(\mathbb{R})$, Comm. Algebra, to appear.

[14] N. Koblitz, Introduction to Elliptic Curves and Modular Forms, Springer, 1984.

[15] S. Lang, Algebraic Number Theory, Springer, 1994.

[16] —, Elliptic Functions, Springer, 1987.

[17] R. Rankin, Modular Forms and Functions, Cambridge Univ. Press, Cambridge, 1977.

[18] B. Schoeneberg, Elliptic Modular Functions, Springer, 1973.

[19] G. Shimura, Introduction to the Arithmetic Theory of Automorphic Functions, Publ. Math. Soc. Japan 11, Princeton, 1971.

[20] —, On modular forms of half-integral weight, Ann. of Math. 97 (1973), 440-481.

[21] J. Silverman, Advanced Topics in the Arithmetic of Elliptic Curves, Springer, 1994.

Department of Mathematics

Korea Advanced Institute of Science and Technology

Taejon 305-701, South Korea

E-mail: hkj@math.kaist.ac.kr

jkkoo@math.kaist.ac.kr

Received on 10.11 .1998

and in revised form on 16.12.1999 